\title{
Regulatory role of DEPTOR-mediated cellular autophagy and mitochondrial reactive oxygen species in angiogenesis in multiple myeloma
}

\author{
JIZHEN WANG ${ }^{1}$, JUNMIN CHEN $^{1}$, DONGBIAO QIU ${ }^{2}$ and ZHIYONG ZENG ${ }^{1}$ \\ Departments of ${ }^{1}$ Hematology and ${ }^{2}$ Blood Transfusion, The First Affiliated Hospital of \\ Fujian Medical University, Fuzhou, Fujian 350005, P.R. China
}

Received March 27, 2020; Accepted November 25, 2020

DOI: $10.3892 /$ ijmm.2020.4831

\begin{abstract}
DEPTOR, an inhibitor of mammalian target of rapamycin (mTOR), is essential for the survival of multiple myeloma (MM) cells. The expression level of DEPTOR is closely related to the prognosis of patients with MM treated with the antiangiogenic agent thalidomide; however, its role in the regulation of angiogenesis has not yet been elucidated. In the present study, the expression levels of DEPTOR and vascular endothelial growth factor (VEGF), and the microvessel density (MVD) of bone marrow (BM) from patients with MM assessed. DEPTORoverexpression plasmid or CRISPR-associated protein 9 (Cas9) and single guided RNAs (sgRNAs) were used to modulate DEPTOR expression. The DEPTOR-mediated angiogenic effects were assessed using a tube formation assay of human umbilical vein endothelial cells (HUVECs) cultured in the collected conditioned medium from MM cell lines with different expression levels of DEPTOR. It was found that the expression level of DEPTOR negatively correlated with the VEGF level and BM MVD in MM. Autophagic activity was regulated by DEPTOR expression, but was not related to thalidomide-binding protein $\mathrm{CRBN}$, which is required for
\end{abstract}

Correspondence to: Dr Zhiyong Zeng, Department of Hematology, The First Affiliated Hospital of Fujian Medical University, 20 Chazhong Road, Fuzhou, Fujian 350005, P.R. China

E-mail: zengzhiyong049@163.com

Abbreviations: $\mathrm{MM}$, multiple myeloma; $\mathrm{BM}$, bone marrow; DMEM, Dulbecco's modified Eagle's medium; ELISA, enzyme-linked immunosorbent assay; GEO, Gene Expression Omnibus; GSEA, Gene Set Enrichment Analysis; HUVECs, human umbilical vein endothelial cells; IHC, immunohistochemistry; IL-6, interleukin 6; ISS, International Staging System; mTOR, mammalian target of rapamycin; MVD, microvessel density; NF- $\kappa \mathrm{B}$, nuclear factor- $\kappa \mathrm{B}$; PI, propidium iodide; RMA, robust multichip average; ROS, reactive oxygen species; sgRNAs, single guided RNAs; VEGF, vascular endothelial growth factor

Key words: multiple myeloma, DEPTOR, autophagy, mitochondrial reactive oxygen species, angiogenesis thalidomide to play an anti-tumor and antiangiogenic role in MM cells. The disruption of DEPTOR protein decreased cellular autophagy, increased VEGF expression in MM cells, and inhibited the tube formation of HUVECs, while a high expression of DEPTOR exerted the opposite effect. Moreover, targeting DEPTOR also resulted in the production of mitochondrial reactive oxygen species (mtROS), the phosphorylation of nuclear factor- $\kappa \mathrm{B}(\mathrm{NF}-\kappa \mathrm{B})$ and an increase in interleukin 6 (IL-6) secretion. Of note, these effects are fully abrogated by treatment with autophagy activator (SMER28) or mitochondrial-specific antioxidant (Mito-TEMPO). Taken together, the present study demonstrates the role of DEPTOR in the regulation of autophagy/mtROS and subsequent angiogenesis. The results provide a novel mechanism for the further understanding of the therapeutic effects of thalidomide on MM.

\section{Introduction}

In recent 15 years, the overall survival of patients with multiple myeloma (MM) has been significantly prolonged with the clinical application of new agents, including immunomodulatory drugs, proteasome inhibitors and monoclonal antibodies (1-5). Thalidomide was the first of these drugs to exhibit its clinical activity and has been shown to be effective against the disease throughout its stages.

Angiogenesis is an important factor for cancer progression, which promotes tumor growth and metastasis; tumors become necrotic or apoptotic in the absence of vascular support. Vascular endothelial growth factor (VEGF) family members are key factors of angiogenesis, and VEGF expression has been described to play a role in the prognosis of a number of types of cancer, such as colorectal cancer (6), breast cancer (7), non-small cell lung carcinoma (8) and Kaposi's sarcoma (9). However, MM was the first hematological malignancy in which the prognostic relevance of angiogenesis was shown (10). Thalidomide has been proven to exert an anti-angiogenic effect; however, the detailed underlying mechanisms remain unclear.

DEPTOR, an inhibitor of the mammalian target of rapamycin (mTOR), has been found to be overexpressed in MM cells and is essential for their survival $(11,12)$. It has been reported 
that patients with MM with a high DEPTOR expression have good responses to thalidomide-based therapy $(13,14)$. Vascular endothelial cell-expressed DEPTOR regulates endothelial cell activation and angiogenic responses through extracellular signal-regulated kinase (ERK)1 and signal transducer and activator of transcription (STAT)-mediated chemokine expression (15). In addition, endothelial cell-specific DEPTOR deficiency increases CD31, hypoxia-inducible factor (HIF)- $1 \alpha$ and VEGF expression, which promotes angiogenesis (16). However, whether DEPTOR can regulate the angiogenesis of $\mathrm{MM}$ cells and the associated underlying mechanisms have not yet been elucidated.

A previous study by the authors demonstrated that the knockdown of DEPTOR by siRNA also inhibited autophagy in MM cells (17). Basal autophagy is necessary for cellular; housekeeping; to eliminate damaged organelles, such as depolarized mitochondria due to reactive oxygen species (ROS) $(13,18,19)$. Mitochondrial ROS (mtROS) are involved in the signal transduction pathways leading to nuclear factor $\kappa \mathrm{B}(\mathrm{NF}-\kappa \mathrm{B})$ activation $(20-22)$. NF- $\kappa \mathrm{B}$ activation plays a dominant role in the secretion of interleukin (IL)-6, which stimulates the secretion of VEGF (21). As mentioned above, it was hypothesized that DEPTOR-mediated cellular autophagy and mitROS may play pivotal regulatory roles in angiogenesis in MM. The analysis described herein may be helpful for the further understanding of the mechanisms underlying the regulatory effects of DEPTOR on angiogenesis and thalidomide for the treatment of MM.

\section{Materials and methods}

Validation of DEPTOR expression in MM based on the Oncomine database. Oncomine (https://www.oncomine.org) is an online database containing previously published and publicly available microarray data. The Oncomine database Wwase used to validate the mRNA expression of DEPTOR in MM cell lines. The DEPTOR gene was queried in the database, and the results were filtered by selecting MM and Cancer vs. Cancer Analysis. Statistical analysis was conducted using Oncomine algorithms.

Overview of the potential pathways of DEPTOR in regulating the biological role of MM cells using microarray profiles from patients with MM. The microarray dataset GSE24080 of 559 patients with MM (23) (Affymetrix Human Genome U133 2.0 Array) was obtained from the Gene Expression Omnibus (GEO) database. The raw array data (.CEL files) were pre-processed using the Robust Multichip Average (RMA) algorithm (24). Microarray annotation information was used to match probes with the corresponding genes. If multiple probes matched a single gene, the probes with the highest quartile range were selected, as previously reported (25). To further investigate the possible pathways of DEPTOR involved in regulating the biological characteristics of myeloma cells, KEGG-related Gene Set Enrichment Analysis (GSEA) analysis was conducted on the on GSE24080 dataset by the gseKEGG function of the clusterProfiler package in R (26).

Cell lines and primary cells. The human MM cell lines, RPMI8226, MM.1S and U226, were obtained from the American Type Culture Collection (ATCC) and grown in
RPMI-1640 medium (HyClone; Thermo Fisher Scientific, Inc.) containing $10 \%$ heat-inactivated fetal calf serum (Biological Industries). Human umbilical vein endothelial cells (HUVECs) and the 293-FT cell line were obtained from the China Center for Type Culture Collection and grown in Dulbecco's modified Eagle's medium (DMEM) (HyClone; Thermo Fisher Scientific, Inc.) containing 10\% heat-inactivated fetal calf serum (Biological Industries). Bone marrow (BM) aspiration and biopsy specimens were obtained from patients newly diagnosed with MM according to diagnostic criteria (27). Primary plasma cells were isolated from BM specimens using CD138 ${ }^{+}$ magnetic-activated cell sorting (MACS; Miltenyi Biotec $\mathrm{GmbH})$. A total of 22 patients were used in the present study and samples were collected at the First Affiliated Hospital of Fujian Medical University from October, 2015 to June, 2018. Approval for the study was obtained from the Ethics Committee of the First Affiliated Hospital of Fujian Medical University. Informed consent was obtained in accordance with the Declaration of Helsinki.

Assessment of BM microvessel density (MVD). BM MVD was estimated as previously described (28). Immunostaining for CD34 was used to evaluate the BM MVD. BM specimens used in the present study were fixed in $10 \%$ formaldehyde overnight and embedded in paraffin. Thin slides of $4 \mu \mathrm{m}$ thickness were cut and subjected to immunohistochemistry (IHC) for CD34 (clone My10; 1:50; ab245689, Abcam) for $32 \mathrm{~min}$ at room temperature. Immunoreactivity was detected with goat anti-rabbit immunoglobulins H\&L (HRP) (1:200; ab6721, Abcam). The reaction was revealed with diaminobenzidine (DAB) and counterstained with Harris' hematoxylin (Dako; Agilent Technologies, Inc.) for $30 \mathrm{~min}$ at room temperature.

The staining was evaluated by 2 independent pathologists under a light microscope (Olympus Corporation). Each slide was scanned at x100 magnification to determine 3 'hotspots', defined as areas with the maximum number of microvessels. Microvessels were counted in each of the 3 hotspots at $x 400$ magnification. Large vessels and vessels in the periosteum or bone were excluded. Areas of staining with no discrete breaks were counted as a single vessel. The presence of a lumen was not required. MVD was estimated by determining the average number of vessels in each of the 3 hotspots.

Cell treatment. Rapamycin (100 nM, Shanghai Shenggong Bioengineering Service Co., Ltd.) was added to the RPMI8226 and MM.1S cells for 24 h. 3-MA (20 $\mu$ M, Sigma-Aldrich; Merck KGaA) was added to the U226 cells followed by culture for $24 \mathrm{~h}$. SMER28 $(10 \mu \mathrm{M}$, Beyotime Institute of Biotechnology) and Mito-TEMPO (10 $\mu \mathrm{M}$, Sigma-Aldrich; Merck KGaA) were added to the RPMI8226 and MM.1S cells followed by culture for $24 \mathrm{~h}$ with DEPTOR inhibition. Thalidomide (Sigma-Aldrich; Merck KGaA) was added to the RPMI8226 and MM.1S cells and cultured for $24 \mathrm{~h}$, with or without DEPTOR inhibition.

Recombinant lentiviral vector construction and infection of cells. Lentiviral vectors harboring Cas9 and sgRNA (Shanghai GeneChem Co., Ltd.) were constructed for the transfection of the MM cells. The sgRNAs targeting the DEPTOR gene were designed using an online tool (http://crispr.mit.edu/). 
The sequences of the sgRNAs were as follows: sgRNA1, GAG TGGCGGGGCGCAGCAAA; sgRNA2, CAAGTATGAGCG CACCTTCA; sgRNA3, TCAGAATGAACTTCCGGCGG; and control sgRNA, CGCTTCCGCGGCCCGTTCAA. The sgRNAs were packaged into lentiviruses, which were named DEP1, DEP2, DEP3 and GFP, respectively.

According to the manufacturer's protocol, lentiviruses were transduced into RPMI8226 (multiplication of infection, MOI=100) and MM.1S (MOI=66) cells with HitransGP (cat. no. REVG005; Shanghai Genechem Co., Ltd.) for 5 days. The cells were then tested for the DEPTOR mutation using the Cruiser ${ }^{\mathrm{TM}}$ Enzyme (Genloci Biotechnologies Inc.) and tested for DEPTOR protein disruption by western blot analysis. Sanger sequencing was performed to confirm the presence of a mutation in the desired part of the genome using the forward primer for DEPTOR sgRNA1, 5'-CTGCCTACC CATAGGGATTCC-3'; sgRNA2, 5'-CTTTGCTGTAGTACT TCATGG-3'; and sgRNA3, 5'-TTTCTCCCCAGTGTCCAA CAAG-3'. The U226 cells were transfected with a DEPTOR overexpression vector (GV143-DEPTOR, Genechem Co., Ltd.) at the concentration of $20 \mu \mathrm{g} / \mathrm{ml}$ in serum-free medium by electroporation. After $48 \mathrm{~h}$, the protein levels of the corresponding genes in transfected U226 cells were detected by western blot analysis. The condition medium derived from the above-mentioned MM cell lines with different expression levels of DEPTOR were collected and centrifuged at $360 \mathrm{x} \mathrm{g}$ for $5 \mathrm{~min}$ at room temperature and stored at $-80^{\circ} \mathrm{C}$.

Cell growth assay. A Cell Counting kit (CCK)-8 assay (Beyotime Institute of Biotechnology, Inc.) was used to assess cell growth, according to the manufacturer's instructions. Briefly, $1 \times 10^{4}$ cells $/ 100 \mu 1$ were seeded into 96 -well plates and cultured for different periods of time $(0,24,48,72,96,120$, 144 and $168 \mathrm{~h}$ ). For the last $4 \mathrm{~h}$ of culture, the cells were incubated with $10 \mu \mathrm{l}$ of CCK- 8 at $37^{\circ} \mathrm{C}$ for $45 \mathrm{~min}$. The absorbance at $450 \mathrm{~nm}$ was measured using an automated microplate reader (Tecan Infinite F50; Tecan Group, Ltd.) to detect metabolically intact cells.

Tube formation assay. Matrigel (100 $\mu \mathrm{l}$; BD Biosciences) was poured into 96 -well plates and then incubated at $37^{\circ} \mathrm{C}$ for $45 \mathrm{~min}$. HUVECs were collected and resuspended in the previously collected conditioned medium at $2 \times 10^{5} / \mathrm{ml}$, and $100 \mu \mathrm{l}$ was seeded on the Matrigel and cultured for $8 \mathrm{~h}$ at $37^{\circ} \mathrm{C}$. The morphological changes of the cells from 3 randomly chosen fields were observed and photographed using a light microscope (Olympus Corporation) at x100 magnification. The number of tube-like structures was measured using ImageJ software version 1.46 (National Institutes of Health).

Dual-luciferase reporter assay. RPMI8226 and MM.1S cells were seeded in 24 -well plates at $5 \times 10^{5} / \mathrm{ml}$. The cells were transfected with the pNFкB-TA-luc reporter plasmid (Beyotime Institute of Biotechnology) at the concentration of $2 \mu \mathrm{g} / \mathrm{ml}$ and the Renilla luciferase-expressing plasmid as an internal control using Lipofectamine 2000 for $48 \mathrm{~h}$ (Lipo2000, Invitrogen Inc.; Thermo Fisher Scientific, Inc.), according to the manufacturer's instructions. A total of 4 groups of cells were prepared: The negative control group, positive control group (TNF- $\alpha$ was used as a NF- $\kappa \mathrm{B}$ activator), and test groups treated with lentivirus GFP and DEP3 for $48 \mathrm{~h}$ before harvesting. Firefly luciferase activities were analyzed using the dual-luciferase reporter assay kit (Beyotime Institute of Biotechnology).

Enzyme-linked immunosorbent assay (ELISA). The quantitative evaluation of IL-6 and VEGF in cell culture supernatants was carried out using ELISA. The cell-free supernatants were collected from cell cultures and centrifuged at $360 \mathrm{x} \mathrm{g}$ for $5 \mathrm{~min}$ at $20^{\circ} \mathrm{C}$. The concentration of secreted VEGF proteins was determined using an ELISA kit (Neobioscience), according to the manufacturer's instructions.

DAPI and MitoSOX staining. The cells were incubated with DAPI $(1 \mu \mathrm{g} / \mathrm{ml}$, Beyotime Institute of Biotechnology) for $12 \mathrm{~h}$, stained with the MitoSOX dye ( $5 \mu \mathrm{M}$; MitoSOX Red Reagent, Invitrogen Inc.; Thermo Fisher Scientific, Inc.) at $37^{\circ} \mathrm{C}$ for $30 \mathrm{~min}$, and fixed with $4 \%$ paraformaldehyde for $20 \mathrm{~min}$. After washing with PBS, the nuclear morphology of the cells and mtROS staining were examined using a fluorescence microscope (Olympus Corporation).

Flow cytometry. For mtROS measurements, the cells were stained with the MitoSOX Red Reagent ( $5 \mu \mathrm{M}$; Invitrogen Inc.; Thermo Fisher Scientific, Inc.) at $37^{\circ} \mathrm{C}$ for $30 \mathrm{~min}$. The stained cells were analyzed for fluorescence using a flow cytometer (FACSCalibur, BD Biosciences). The data were analyzed using Flowjo software (Flowjo 10.0).

Cell apoptosis assay was performed using flow cytometry with propidium iodide (PI) and Annexin V-APC staining (APC Annexin V Apoptosis Detection kit with PI; 640932, BioLegend, Inc.). The cells were harvested, washed twice with PBS, resuspended in staining buffer, and stained with Annexin V-APC/PI, according to the manufacturer's instructions. The cells were analyzed using a flow cytometer (FACSCalibur, BD Biosciences).

Monodansylcadaverine (MDC) staining. The autofluorescent compound, MDC [Sangon Biotech (Shanghai) Co., Ltd.] is a commonly used selective fluorescent marker for autophagic vacuoles. The cells were plated on glass coverslips and probed with a 1:1,000 dilution of MDC in staining buffer (Cell-Based Assay Buffer Tablet dissolved in water). Following $10 \mathrm{~min}$ of incubation at $37^{\circ} \mathrm{C}$, the cells were washed twice with assay buffer in the dark. A fluorescence microscope (Olympus Corporation) was used immediately after MDC staining.

Western blot analysis. For protein extraction, the cells were lysed in RIPA buffer (Beyotime Biosciences) containing the protease inhibitor PMSF. The isolation of mitochondrial and cytosolic proteins was performed using the Mitochondria/Cytosol Fractionation kit (Beyotime Institute of Biotechnology). Cytoplasmic and nuclear protein extracts were prepared using the NE-PER ${ }^{\mathrm{TM}}$ Nuclear and Cytoplasmic Extraction Reagents (Thermo Fisher Scientific, Inc.), according to the manufacturer's instructions.

Protein quantification were detected using spectrophotometer (DS-11, DeNovix). A total of $80 \mu \mathrm{g}$ protein/lane were separated by SDS-PAGE on 8-16\% Tris-glycine gels (Beyotime Institute of Biotechnology), transferred to polyvinylidene 
difluoride membranes (Millipore corp., Billerica, MA, USA). The membranes were then blocked for $1 \mathrm{~h}$ at room temperature with non-fat dry milk in TBST (Bio-Rad Laboratories, Inc.). The membranes were incubated with antibodies against LC3 (1:1,000, ab51520, Abcam), Beclin 1 (1:1,000, ab62557, Abcam), DEPTOR (1:1,000, ab244394, Abcam), VEGF (1:1,000, AV202, Beyotime Institute of Biotechnology), phospho-p65 (p-p65, 1:1,000, ab109458, Abcam), p65 (1:1,000, ab16502, Abcam), phospho-p50 (p-p50, 1:1,000, 710460, Invitrogen; Thermo Fisher Scientific, Inc.), p50 (1:1,000, 710450, Invitrogen; Thermo Fisher Scientific, Inc.), IкB- $\alpha$ (1:1,000, ab7217, Abcam), cytochrome $c$ (Cyt $c ; 1: 1,000$, ab90529, Abcam), cytochrome $c$ oxidase subunit IV (COX IV; 1:1,000, ab16056, Abcam), CRBN (1:2,000, ab98992, Abcam), Lamin B (1:1,000, 66095-1, ProteinTech Group, Inc.) and $\beta$-actin (1:2,000, ab8226, Abcam) at $4^{\circ} \mathrm{C}$ overnight. Following 3 washes with TBS-T for $10 \mathrm{~min}$, a peroxidase-conjugated secondary antibody $(1: 10,000$, A0216/A0208, Beyotime Institute of Biotechnology) was incubated with the membranes for $2 \mathrm{~h}$ at room temperature. Signals were detected by the ECL detection system (Beyotime Institute of Biotechnology). The results were analyzed quantitatively by densitometry using software ImageJ software version 1.46 (National Institutes of Health).

Purpose proteins and loading control with similar molecular weight were produced from the same western blot membrane. Purpose protein detected by the ECL detection system, incubated with stripping buffer for approximately 30 min under constant agitation; following 3 washes with TBS-T for $10 \mathrm{~min}$ and then blotted with antibodies against loading control ( $\beta$-actin and Lamin B). Following 3 washes with TBS-T for $10 \mathrm{~min}$, a peroxidase-conjugated secondary antibody (Beyotime Institute of Biotechnology) was incubated for $2 \mathrm{~h}$ at room temperature. Signals were detected by the ECL detection system (Beyotime Institute of Biotechnology). The results were analyzed quantitatively by densitometry using ImageJ software version 1.46 (National Institutes of Health).

Statistical analysis. All experiments were performed in triplicate. The data are presented as the means \pm standard deviation (SD). Differences were analyzed using the Student's t-test or one-way ANOVA with the Tukey's HSD post hoc test, as appropriate. The associations between the expression of DEPTOR and the expression of VEGF and BM MVD and in patients with MM were assessed by Pearson's correlation analysis. GraphPad 5.0 (GraphPad Software Inc.) was used for statistical analyses. A P-value $<0.05$ was considered to indicate a statistically significant difference.

\section{Results}

Validation of DEPTOR expression in MM cell lines using the Oncomine database. The mRNA expression levels of DEPTOR in MM were explored using the Oncomine database. According to the results of the Cancer vs. Cancer Analysis, 3 datasets (Barretina CellLine, Garnett CellLine and Wooster CellLine) were identified that contained DEPTOR mRNA expression data obtained from different tumor cell lines. As shown in Fig. 1A-C, 2 datasets (Barretina CellLine and Garnett CellLine) demonstrated that DEPTOR mRNA expression was significantly higher in MM than in the majority of other cancers (including bladder cancer, brain and central nervous system cancer, breast cancer, cervical cancer, colorectal cancer, esophageal cancer, gastric cancer, head and neck cancer, kidney cancer, leukemia, liver cancer, lung cancer, lymphoma, melanoma, ovarian cancer, pancreatic cancer, prostate cancer and sarcoma); however, one dataset (Wooster CellLine) revealed no differences between MM and other types of cancer. The meta-analysis of these 3 datasets still suggested that DEPTOR was overexpressed in MM compared with other types of cancer (Fig. 1D).

Overview of potential pathways of DEPTOR involved in regulating the biological pathway of MM cells using microarray profiles. To investigate the possible pathways of DEPTOR involved in the regulation of MM cells, KEGG-related GSEA analysis was conducted on the GSE24080 dataset using the gseKEGG function of the clusterProfiler package in R. As shown in Fig. 1E, the main enriched pathways closely related to cancer development included protein processing in the endoplasmic reticulum, oxidative phosphorylation, autophagy, ubiquitin-mediated proteolysis, the mTOR signaling pathway and the VEGF signaling pathway.

DEPTOR expression is negatively related to VEGF expression and $B M M V D$ in $M M$. The mRNA expression levels of VEGFA in MM were also explored using the Oncomine database. As shown in Fig. S1A-C, VEGFA mRNA expression was significantly higher in MM than in the majority of other types of cancer in the Barretina CellLine and Garnett CellLine databases; however, the Wooster CellLine database did not reveal any marked differences between MM and other types of cancer. The meta-analysis of these 3 datasets still suggested that VEGFA was overexpressed in MM compared with other types of cancer (Fig. S1D).

Western blot analysis was used to examine the expression levels of DEPTOR and VEGF in different MM cell lines (RPMI8226, MM.1S and U226). The results revealed that the RPMI8226 cells had the highest expression of DEPTOR and the lowest expression of VEGF, while the MM.1S cells had an intermediate expression of DEPTOR and VEGF, and the U266 cells had the lowest expression of DEPTOR and the highest expression of VEGF (Fig. 1F and G).

In the cohort of 22 patients newly diagnosed with MM, the expression patterns of DEPTOR and VEGF in primary MM cells isolated by MACS were validated by western blot analysis. The 22 patients with MM included 1 patient with the International Staging System (ISS) I, 7 with ISS II and 14 with ISS III. The median age was 65 years (range, $46-89$ years). In total 12 patients were male and 10 were female. Western blot analysis and Pearson's correlation analysis revealed that the expression of DEPTOR negatively correlated with the expression of VEGF (Pearson's r=-0.78, Fig. 1H and I). The MVD in BM specimens of corresponding patients was investigated by IHC with an anti-CD34 antibody (Fig. S2). The median BM MVD was 167 (range, 9-412 vessels $/ \mathrm{mm}^{2}$ ). Using the median relative expression among the 22 patients $(0.12$, which corresponds to the average value between the 11 and 12th patients) of DEPTOR as a cut-off value, the patients were divided into 2 groups, those with a high and low DEPTOR expression. The MVD in the high expression DEPTOR group 
A Barretina CellLine statistics

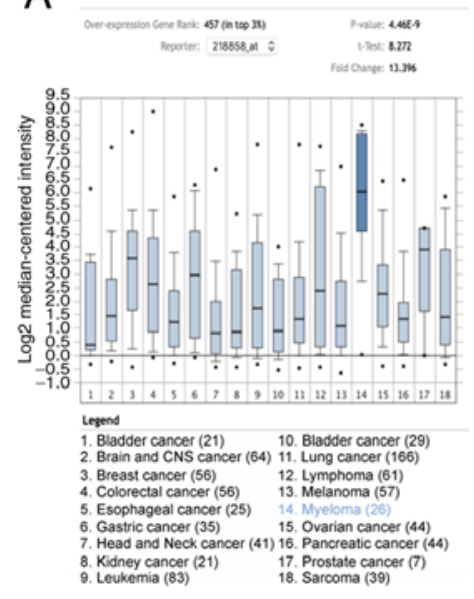

B Garnett CellLine statistics
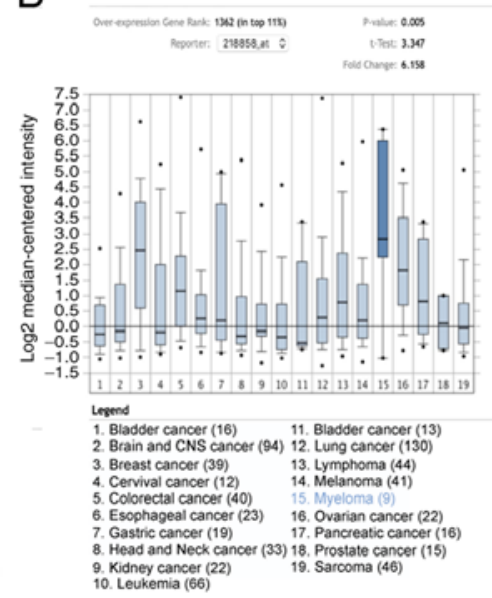

C Wooster CellLine statistics

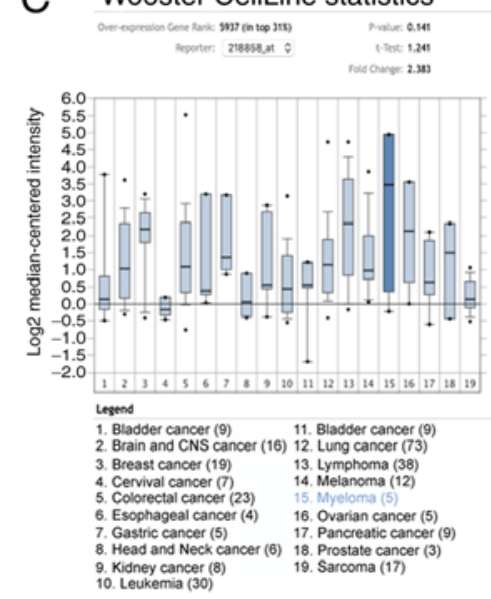

D

Comparison of DEPDC 6 across 3 analyses Over-expression

\begin{tabular}{|c|c|c|c|}
\hline Median Rank & p-Value & Gene & \\
\hline \multirow[t]{2}{*}{1362.0} & 0.005 & DEPOC6 & \\
\hline & & & \begin{tabular}{|l|l|l|}
1 & 2 & 3 \\
\end{tabular} \\
\hline
\end{tabular}

Legend

1. Cancer Type: Myeloma 3. Cancer Type: Myeloma Barretina CellLine, Wooster CellLine, Not Published, 2008

2. Cancer Type: Myeloma

Garnett Celline, Nature, 2012

\begin{tabular}{l}
151025251051 \\
1000 \\
\hline
\end{tabular}

Rank for a gene is the median rank for that gene across each of the analyses. p-value for a gene is its p-value for the median-ranked analysis.

E
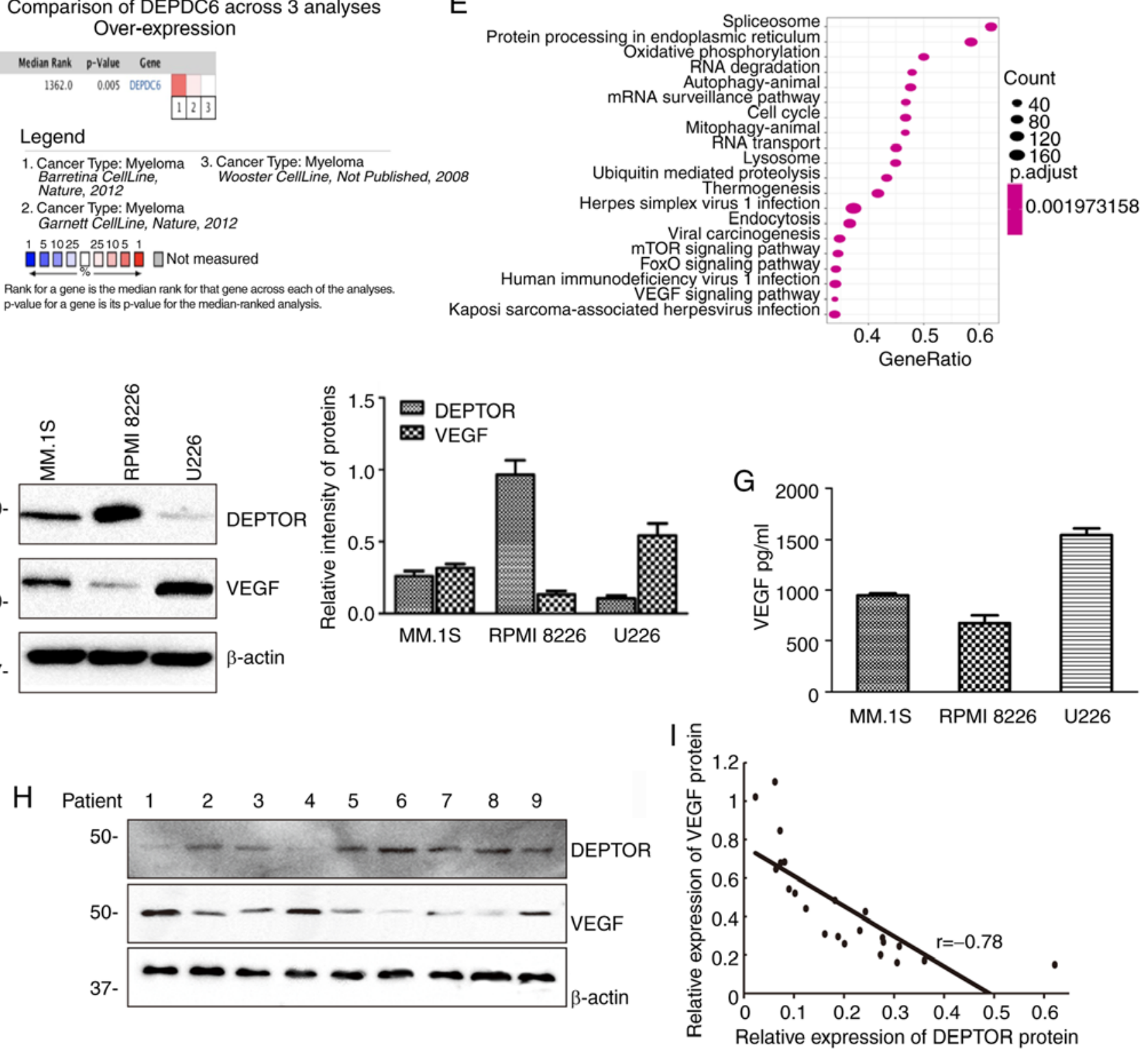

Figure 1. Continued.

was significantly lower than in the low expression DEPTOR group $\left(96.9 \pm 22.0\right.$ vessels $/ \mathrm{mm}^{2}$ vs. $281.2 \pm 28.6$ vessels $/ \mathrm{mm}^{2}$, respectively; $\mathrm{P}<0.001$; Fig. $1 \mathrm{~J}$ ). The expression of DEPTOR negatively correlated with MVD (Pearson's r=-0.76) (Fig. 1K). 
J

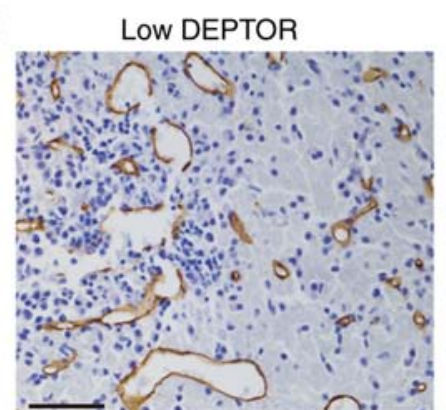

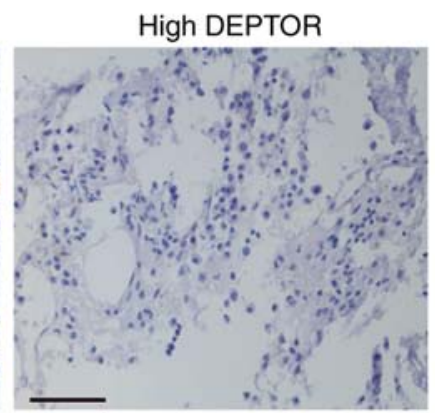

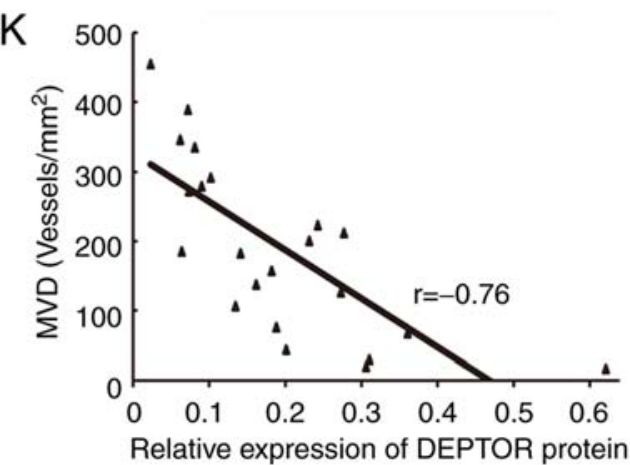

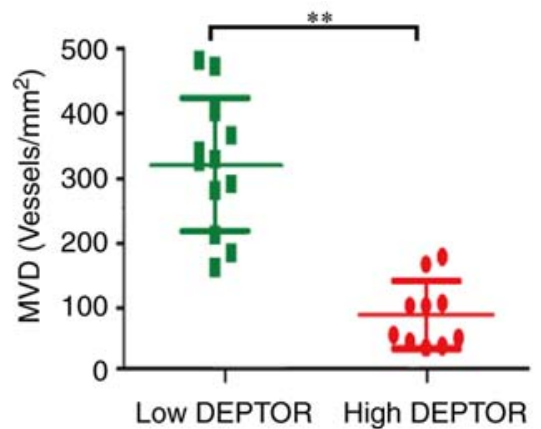

Figure 1. Association between DEPTOR and VEGF expression levels in MM. (A-C) Expression of DEPTOR in 3 datasets (Barretina CellLine, Garnett CellLine and Wooster CellLine) from the Oncomine database. The asterisks (*) indicate statistical significance. (D) Meta-analysis of the 3 Oncomine datasets on the DEPTOR expression difference between MM and other cancers. (E) Potential pathways of DEPTOR involved in the regulation of MM cells identified by Gene Set Enrichment Analysis. (F) Western blot analysis of DEPTOR and VEGF with indicated antibodies from MM cells lines (MM.1S, RPMI8226 and U266). (G) VEGF expression in the conditioned media of myeloma cell lines. (H) Representative western blot analysis of DEPTOR and VEGF from primary MM cells from 9 of 22 patients with newly diagnosed MM. (I) Correlation analysis between DEPTOR and VEGF relative expression levels in 22 patients with newly diagnosed MM. (J) Representative image of MVD with anti-CD34 immunostaining in bone marrow tissues of from patients with newly diagnosed MM with a high or low DEPTOR expression (scale bar, $1 \mathrm{~mm}$ ). Microvessels are stained brown. (K) Correlation analysis between the relative expression level of DEPTOR and bone marrow MVD in 22 patients with newly diagnosed MM. ${ }^{* *} \mathrm{P}<0.01$. VEGF, vascular endothelial growth factor; MM, multiple myeloma; MVD, microvessel density.

CRISPR/Cas9-mediated efficient DEPTOR disruption in MM. The CRISPR/Cas9 system was used to disrupt the protein expression of DEPTOR in the MM cell line, RPMI8226. Lentiviruses (DEP1, DEP2 and DEP3) expressing sgRNA targeting DEPTOR were transfected into RPMI8226 cells (Fig. S3A). At 5 days following transduction, the fragments of the 3 different target sites relevant to DEP1, DEP2 and DEP3 were amplified bu PCR and then digested with Cruiser ${ }^{\mathrm{TM}}$ Enzyme (Fig. S3B). The cleaved bands were observed in the DEP1, DEP2 and DEP3 groups, but not in the control and GFP groups (Fig. S3C), indicating that insertion or deletion mutations (indel) were introduced in the genomes. The levels of DEPTOR protein expression were detected by western blot analysis. The results revealed that there were significant disruptive effects on the DEPTOR protein levels in each sgRNA group compared with the GFP group. Among these, DEP3 displayed the most potent disruptive effect. To further validate DEPTOR gene disruption, the nucleotide sequences of the above PCR products of target DNA in the DEP3 group were analyzed and it was confirmed that the indel mutation was introduced into the genome (Fig. S3D). Therefore, lentivirus DEP3 was used in the subsequent experiments. After the RPMI8226 and MM.1S cells were transfected with lentivirus DEP3, the suppression of DEPTOR abolished cell proliferation over a 7-day culture period (Fig. S3E and F). In addition, the suppression of DEPTOR in RPMI8226 cells induced cell lysis and necrosis from the 5th day (data not shown). This strongly suggests that the high expression of DEPTOR is essential for maintaining myeloma cell survival.

Reduction of DEPTOR expression in MM cells attenuates the effects of thalidomide on proliferation, autophagy and VEGF expression. Based on its antitumor and anti-angiogenic effects, thalidomide was used to treat the $\mathrm{MM}$ cells and was proven to be effective. As shown in Fig. 2A and C, the rate of proliferation of the RPMI8226 and MM.1S cells decreased with the increasing thalidomide concentration and treatment duration. In addition, the expression of DEPTOR was increased with the increasing thalidomide concentration, whereas the expression of VEGF decreased (Fig. 2B and D). These results indicated that DEPTOR expression was negatively associated with the expression of VEGF. The RPMI8226 cells in which DEPTOR expression was suppressed exhibited a marked anti-proliferative response to thalidomide (Fig. 2E). Of note, thalidomide failed to induce the death of DEPTOR-disrupted MM cells (Fig. 2F). Since cell death is not only related to apoptosis, but also to autophagy, the effects of thalidomide on the autophagy of MM cells were further analyzed. MDC staining demonstrated that thalidomide significantly increased the level of autophagy in MM cells, and its effect was suppressed by the autophagy inhibitor, 3-MA (Fig. 2G). Western blot analysis revealed that thalidomide increased DEPTOR and CRBN expression, as well as the expression of autophagy-related proteins (Beclin 1 and LC3 II/I), whereas it inhibited VEGF expression. These 


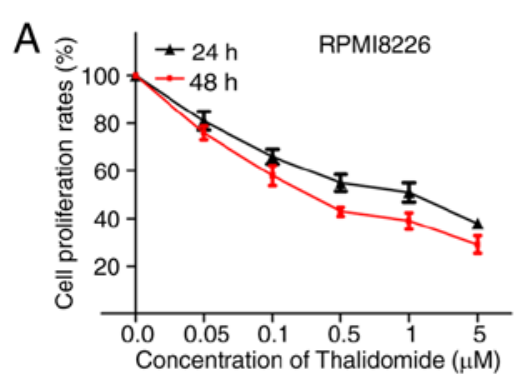

B
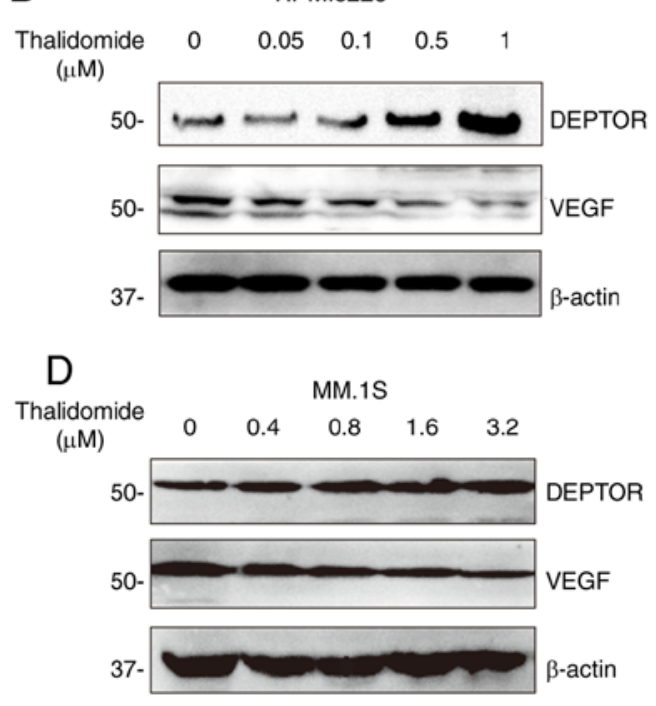
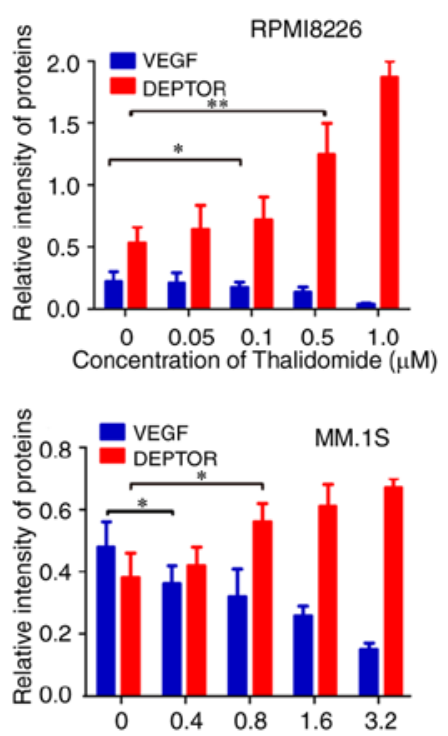

Concentration of Thalidomide $(\mu \mathrm{M})$
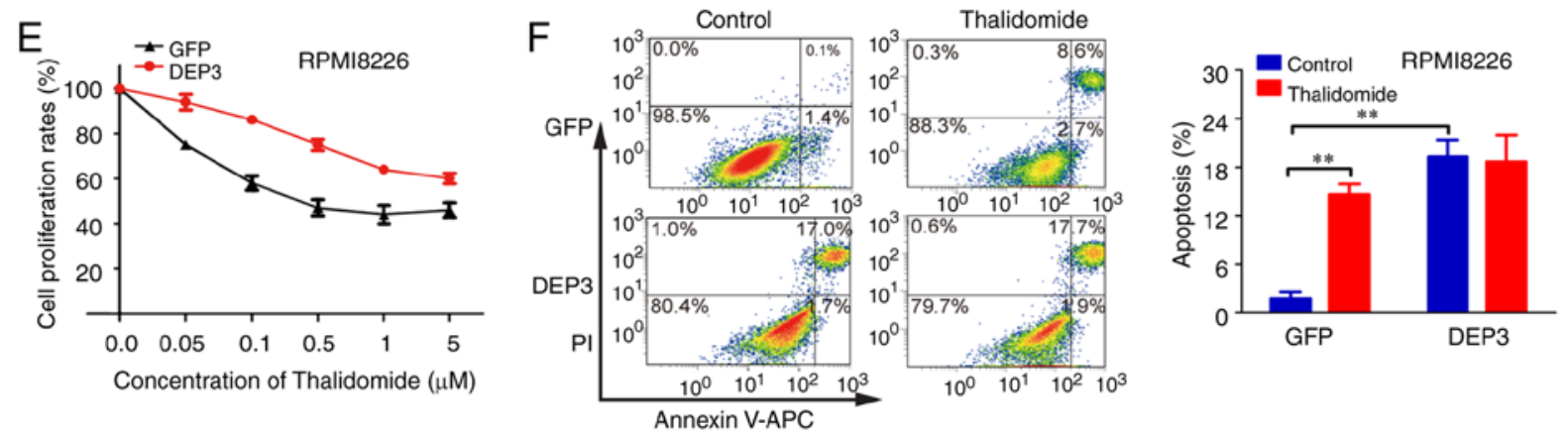

G
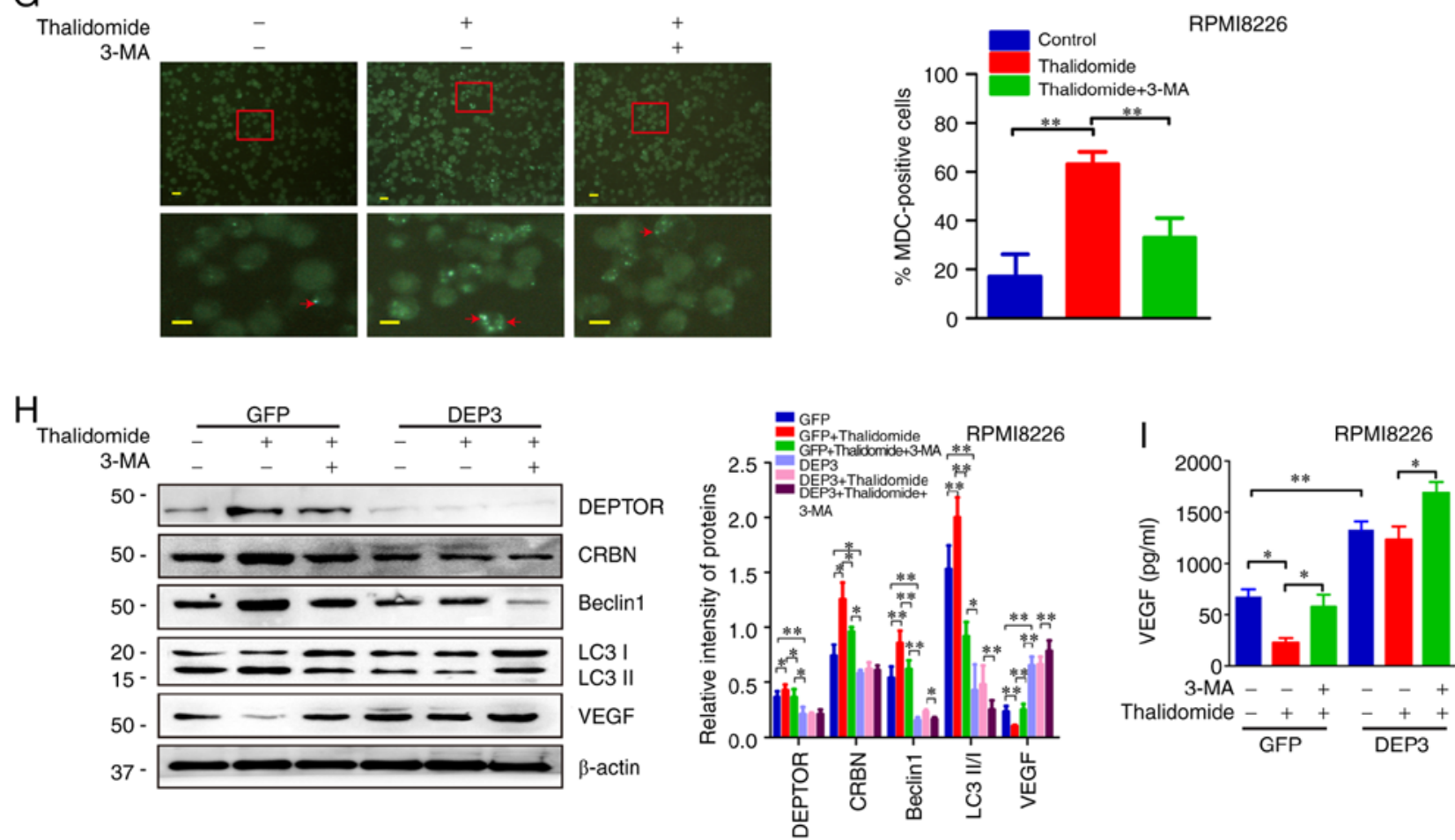

Figure 2. Effect of DEPTOR expression on the antitumor and inhibitory effects of thalidomide on VEGF in MM cells. (A and C) Effect of thalidomide on the proliferation of RPMI 8226 and MM.1S. (B and D) Effects of thalidomide on DEPTOR and VEGF protein expression of RPMI8226 and MM.1S. (E) Effects of thalidomide on the proliferation of RPMI8226 transfected with lentivirus GFP or DEP3. (F) Effects of thalidomide on the apoptosis of RPMI8226 cells transfected with lentivirus GFP or DEP3. (G) Effects of thalidomide and 3-MA on autophagy of RPMI8226 detected by monodansylcadaverine (MDC) staining (scale bar, $10 \mu \mathrm{m}$ ). (H and I) RPMI8226 transfected with lentivirus GFP and DEP3 for 5 days were treated with thalidomide and 3-MA. (H) Cell lysates were examined by western blot analysis for the levels of the indicated proteins. (I) VEGF in culture media was collected and analyzed by ELISA. Error bars indicate the standard deviation from 3 independent experiments. "P<0.05, ${ }^{* *} \mathrm{P}<0.01$. VEGF, vascular endothelial growth factor; MM, multiple myeloma. 
A

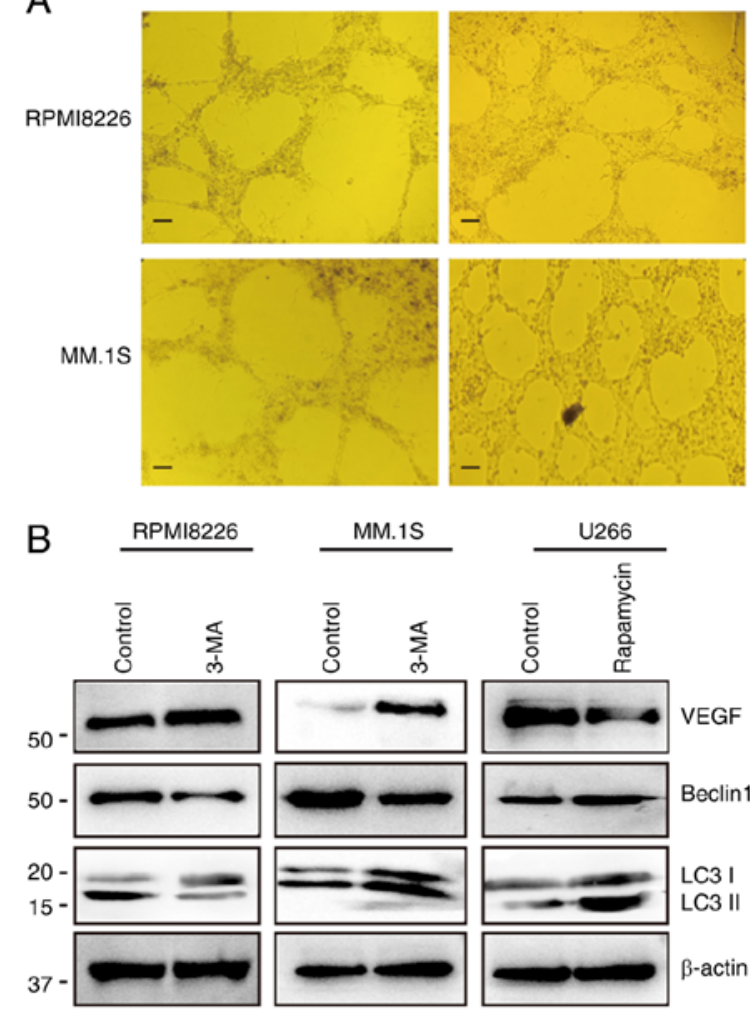

GV143

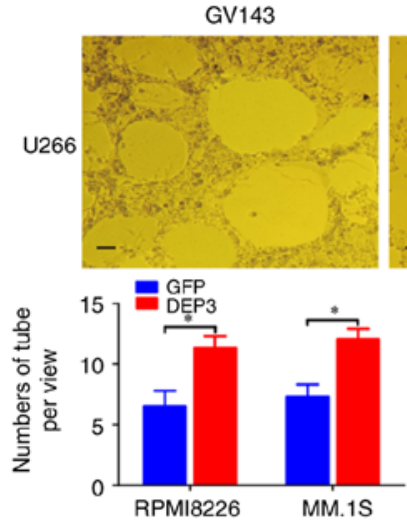

GV143-DEPTOR

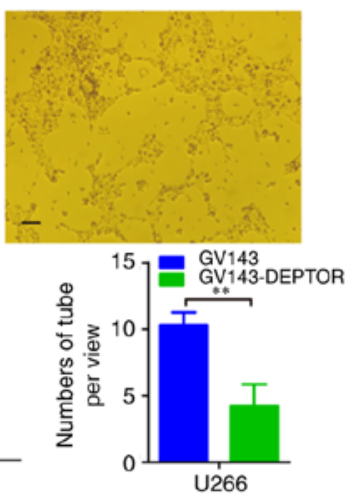

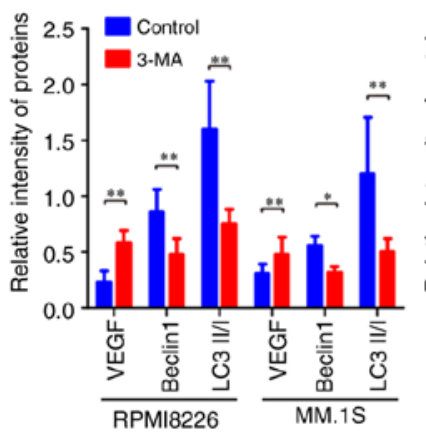
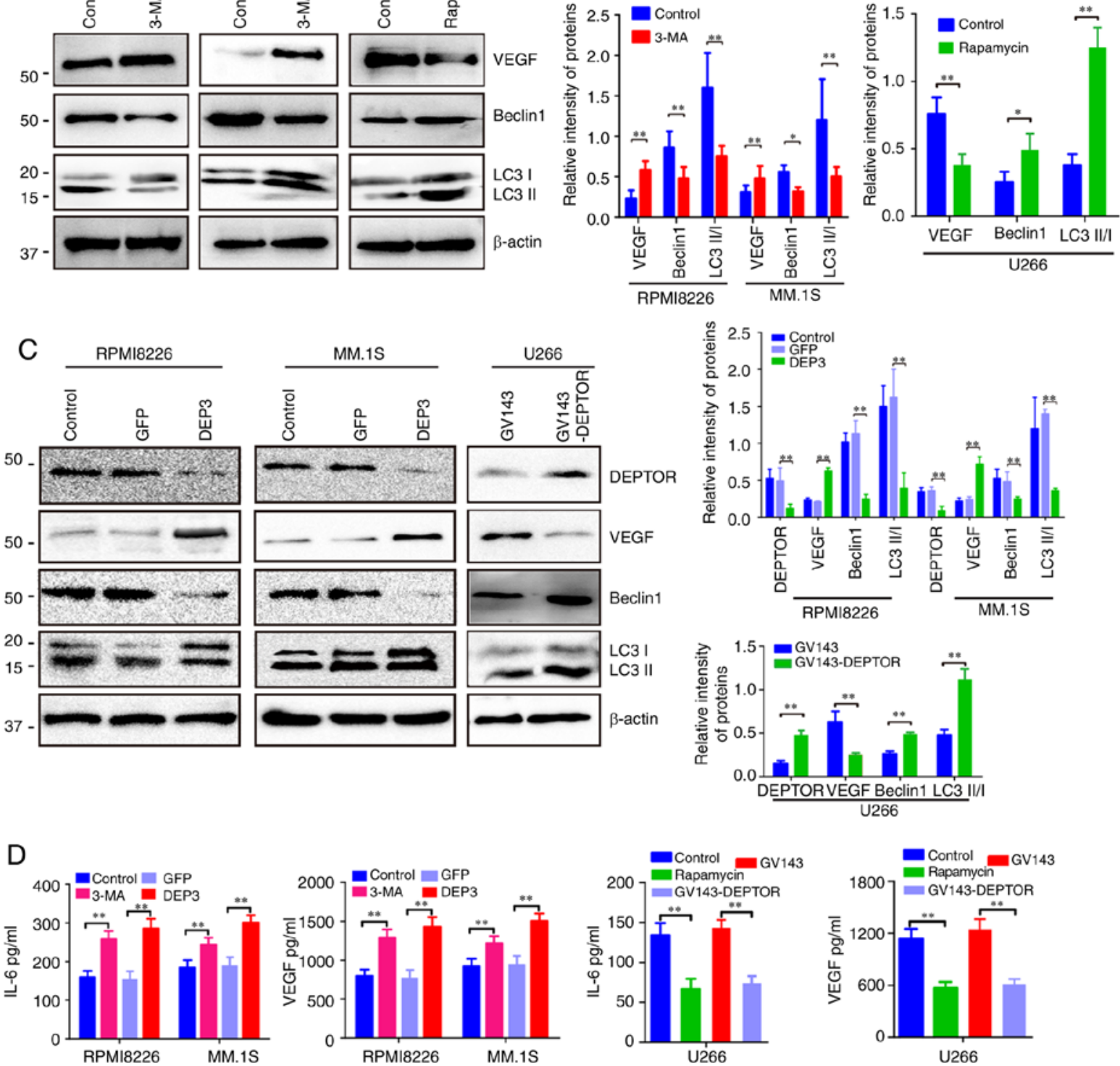

Figure 3. DEPTOR disruption increases autophagy-related angiogenesis in MM cells. The RPMI8226 and MM.1s cells were infected with lentivirus GFP or DEP3 for 5 days; the U226 cells were transfected with blank vector (GV143) or DEPTOR over-expressed vector (GV143-DEPTOR) for 2 days. (A) Their culture media were collected and analyzed for tube formation of HUVECs (scale bar, $10 \mu \mathrm{m}$ ). The numbers of tubes per view are shown as means \pm SD ( $\mathrm{n}=6$ ). (B) RPMI8226 and MM.1S cells were treated with 3-MA $(20 \mu \mathrm{M})$ for $24 \mathrm{~h}$, while U226 cells were treated with rapamycin (100 nM) for 24 h. Cell lysates were examined by western blot analysis for the levels of the indicated proteins. Error bars indicate the standard deviation from 3 independent experiments. (C) RPMI8226 and MM.1S cells were transfected with lentivirus GFP and DEP3 for 5 days, while U266 cells were transfected with GV143 or GV143-DEPTOR for 2 days. Cell lysates were ere examined by western blot analysis for the levels of the indicated proteins. Error bars indicate the standard deviation from 3 independent experiments. (D) IL-6 and VEGF in culture media were collected from cells treated as in (B and C) and analyzed by ELISA. Error bars indicate the standard deviation from 3 independent experiments. ${ }^{*} \mathrm{P}<0.05,{ }^{* *} \mathrm{P}<0.01$. VEGF, vascular endothelial growth factor; MM, multiple myeloma; HUVECs, human umbilical vein endothelial cells. 

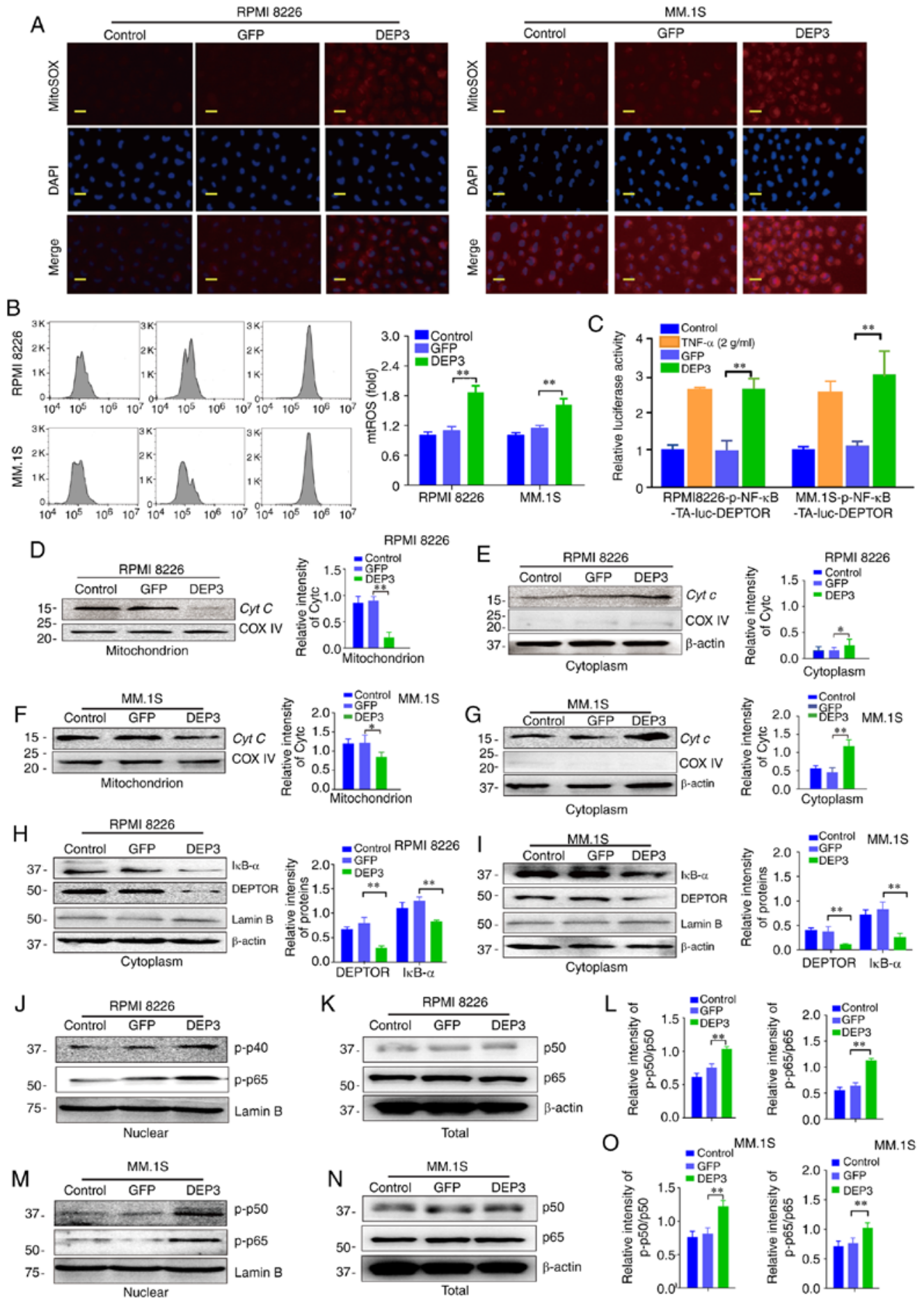

Figure 4. Mitochondrial damage signals in DEPTOR-inhibited RPMI8226 and MM.1S cells. RPMI8226 and MM.1s cells were infected with lentiviral GFP and DEP3 for 5 days. (A and B) The mtROS levels of the RPMI8226 and MM.1S cells in the indicated groups stained with the MitoSOX probe was evaluated using a fluorescence microscope (scale bar, $10 \mu \mathrm{m}$ ) and flow cytometric analysis, respectively. (C) The NF- $\mathrm{kB}$ activity of RPMI8226 or MM.1S cells in the indicated groups was detected by luciferase assays. Cells were co-transfected with pNF-kB-TA-luc with Renilla luciferase reporter (as an internal control) for $24 \mathrm{~h}$ and treated with GFP or DEP3 for $48 \mathrm{~h}$. (D-G) The mitochondrial and cytoplasmic fractions of the cultured cells were separated using the Mitochondria/ Cytosol Fractionation kit. Protein expression of Cyt $c$ in the (D) mitochondria and (E) cytoplasm of the indicated groups for RPMI8226 cells was examined by western blot analysis. Primary antibodies with COXIV and $\beta$-actin was used as the mitochondrial and cytosolic loading control, respectively. Protein expression of Cyt $c$ in the (F) mitochondria and (G) cytoplasm of the indicated groups for MM.1S cells was examined by western blot analysis. Primary antibodies with COXIV and $\beta$-actin was used as the mitochondrial and cytosolic loading control, respectively. Protein expression levels of DEPTOR and IкB- $\alpha$ in the cytoplasm of the indicated groups for (H) RPMI8226 and (I) MM.1S cells were examined by western blot analysis. (J-O). The nuclear and cytoplasmic protein fractions of the cultured cells were isolated using the NE-PER ${ }^{\mathrm{TM}}$ Nuclear and Cytoplasmic Extraction Reagents. Protein expressions of (J) p-p50 and p-p65 in the nuclear, $(\mathrm{K}) \mathrm{p} 50$ and p65 in the whole cell lysates of the indicated groups for RPMI8226 cells was examined by western blot analysis. LaminB and $\beta$-actin was used as the loading control for the nuclear extracts and the whole cell lysates, respectively. (L) Quantification of p-p50 subunit and p-p65 subunit was normalized for total p50 protein and total p65 protein $(\mathrm{J}$ and $\mathrm{K})$, respectively. Protein expressions of $(\mathrm{M}) \mathrm{p}-\mathrm{p} 50$ and p-p65 in the nuclear, (N) p50 and p65 in the whole cell lysates of the indicated groups for MM.1S was examined by western blot analysis, respectively. LaminB and $\beta$-actin was used as the loading control for the nuclear extracts and the whole cell lysates, respectively. (O) Quantification of p-p50 subunit and p-p65 subunit was normalized for total p50 protein and total p65 protein ( $\mathrm{M}$ and N), respectively. Error bars indicate the standard deviation from 3 independent experiments. "P<0.05 compared with GFP group, ${ }^{* *} \mathrm{P}<0.01$ compared with GFP group. Cyt $c$, cytochrome $c$; mtROS, mitochondrial reactive oxygen species; p-p50, phospho-p50; p-p65, phospho-p65. 
A
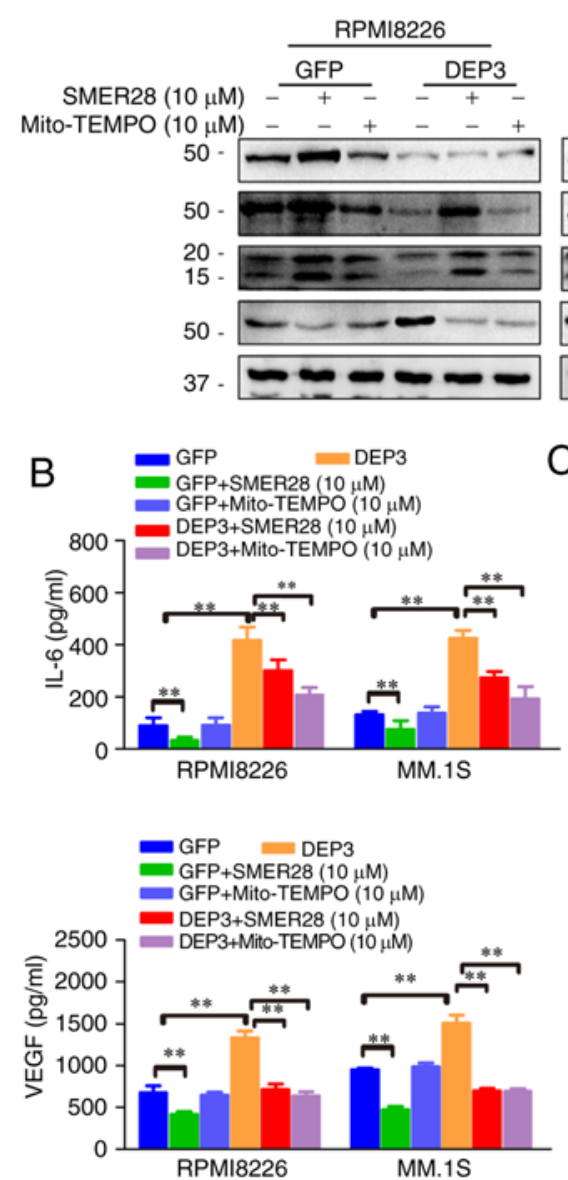
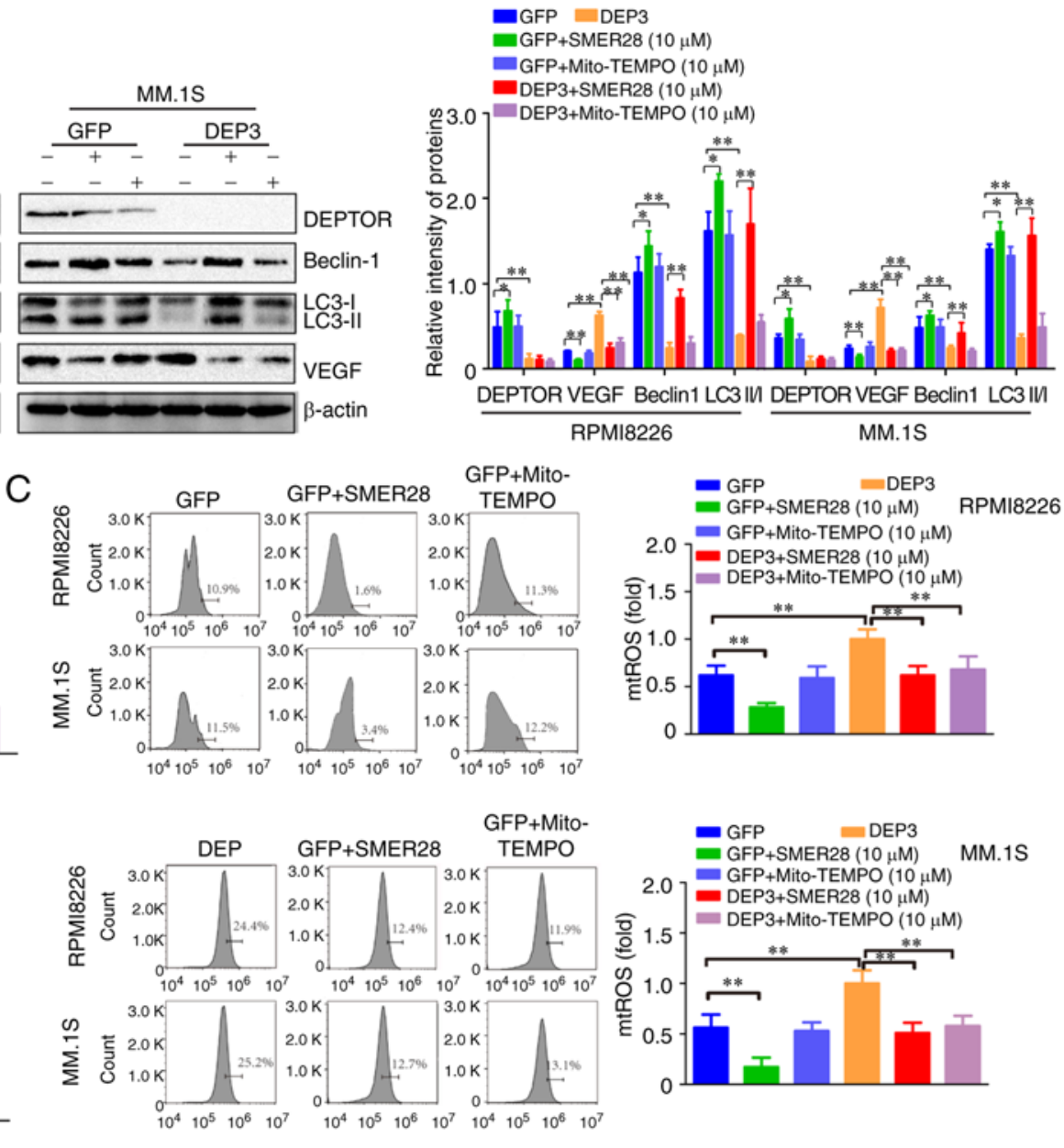

Figure 5. Autophagy inducer and mitochondrial-specific antioxidants reversed the effects of DEPTOR on the angiogenesis of MM cells. RPMI8226 and MM.1S cells were transfected with lentivirus DEP3 for 5 days and exposed to SMER28 (10 $\mu \mathrm{M})$ or Mito-TEMPO (10 $\mu \mathrm{M})$ for $24 \mathrm{~h}$. (A) Cell lysates were examined by western blot analysis for the levels of the indicated proteins. (B) Levels of IL-6 and VEGF in medium supernatant in the indicated groups were examined by ELISA. (C) mtROS levels in the indicated groups stained with the MitoSOX probe were evaluated using flow cytometric analysis. * $\mathrm{P}<0.05$, ${ }^{* *} \mathrm{P}<0.01$. VEGF, vascular endothelial growth factor; MM, multiple myeloma; mtROS, mitochondrial reactive oxygen species.

effects were reversed by 3-MA (Fig. 2H). In the RPMI8226 cells in which DEPTOR expression was disrupted (suppressed), thalidomide had no marked effects on the levels of CRBN, VEGF and autophagy-related proteins (Beclin 1 and LC3 II/I). Furthermore, after the DEPTOR-disrupted RPMI8226 cells were treated with thalidomide and 3-MA, the levels of autophagy-related proteins were further inhibited, while VEGF protein levels and VEGF levels in the culture media were increased (Fig. 2H and I). These data suggested that DEPTOR-regulated autophagy played an important role in the antitumor and anti-angiogenic effects of thalidomide.

Effects of DEPTOR on autophagy and angiogenesis of MM cells. The effects of DEPTOR on angiogenesis in myeloma cell lines were further validated by disrupting or overexpressing DEPTOR. Based on the above-mentioned results of DEPTOR expression levels in MM cell lines, the expression of DEPTOR was suppressed/disrupted in RPMI8226 and MM.1S cells, and overexpressed in U266 cells. The tube formation assay of HUVECs revealed that the RPMI8226 and MM.1S cells in which DEPTOR was inhibited exhibited an increased angiogenesis, while the DEPTOR-overexpressing U266 cells exhibited a suppressed angiogenesis (Fig. 3A). To clarify the effects of DEPTOR on angiogenesis and autophagy, the association between angiogenesis and autophagy in MM was first investigated. As shown in Fig. 3B, when the RPMI8226 and MM.1S cells with a higher autophagic activity were treated with the autophagy inhibitor, 3-MA, their VEGF expression levels increased as autophagic activity decreased. On the other hand, when the U266 cells with a lower autophagic activity were treated with the autophagy inducer, rapamycin, their VEGF expression levels decreased as autophagy activity increased. Furthermore, DEPTOR disruption in RPMI8226 and MM.1S cells decreased the expression of autophagy-related proteins (Beclin-1 and LC3II/LCI) and enhanced VEGF expression (Fig. 3C). The overexpression of DEPTOR in the U266 cells led to the opposite results (Fig. 3C). IL-6 and VEGF levels in the cell supernatant were increased in the RPMI8226 and MM.1S cells following the inhibition of autophagy or the disruption of DEPTOR expression, and were decreased in the U266 cells following the induction of autophagy or the overexpression of DEPTOR (Fig. 3D).

Inhibition of autophagy by DEPTOR disruption activates the $N F-\kappa B$ pathway by damaging mitochondria and promoting the accumulation of mtROS. The present tudy then investigated 


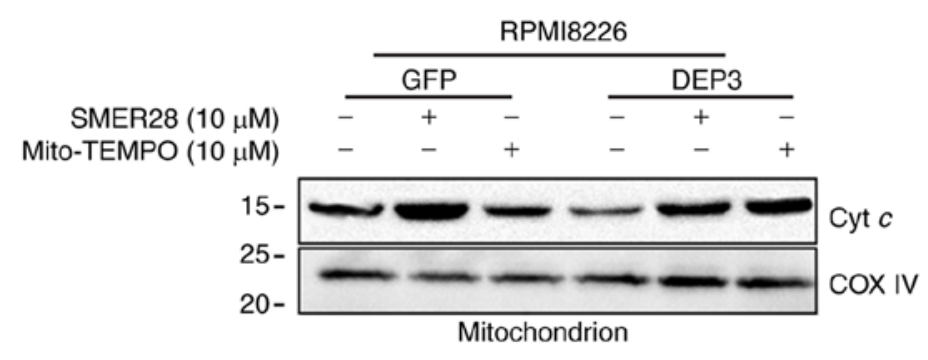

B

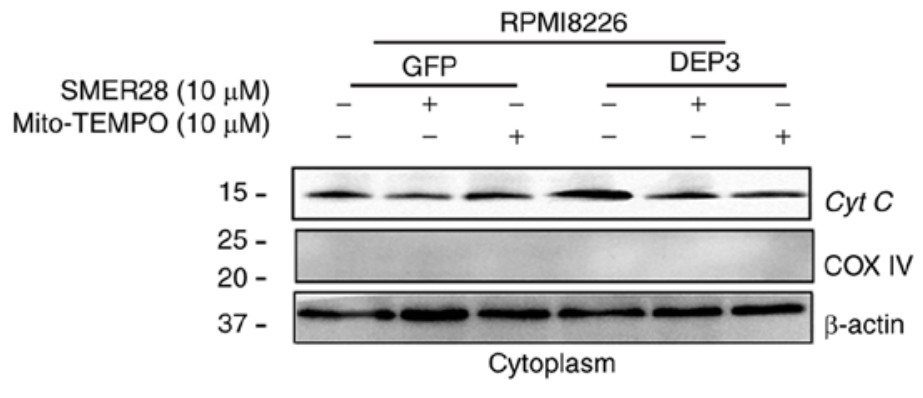

C

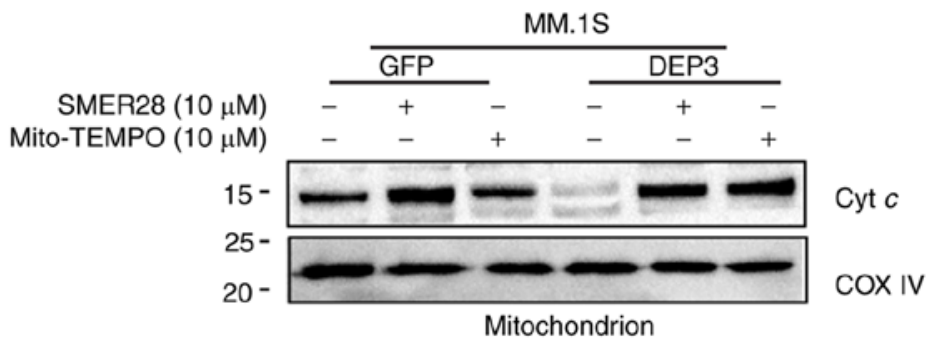

D

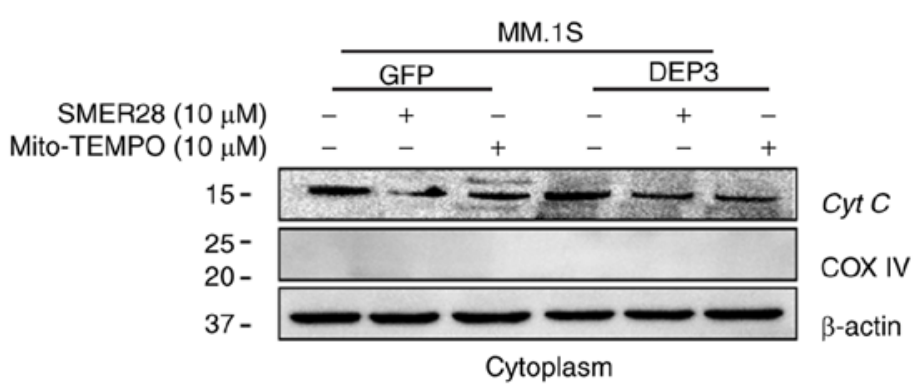

E

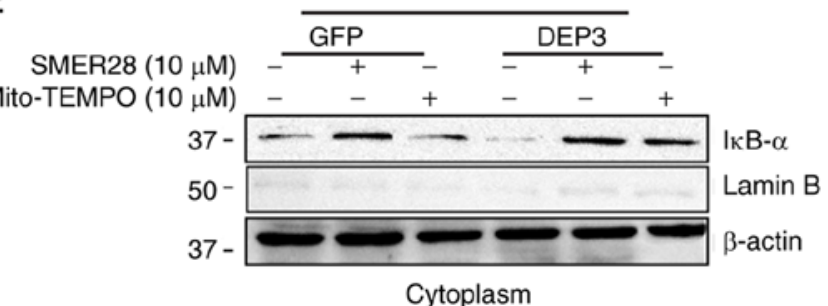

GFP DEP3

GFP+SMER28 $(10 \mu \mathrm{M})$

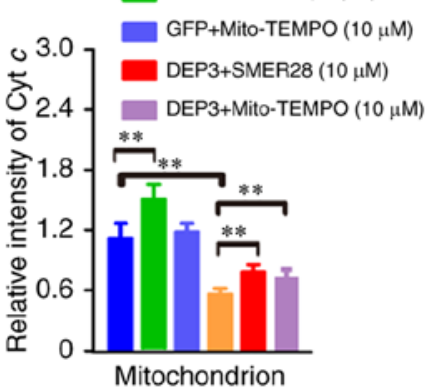

RPMI 8226

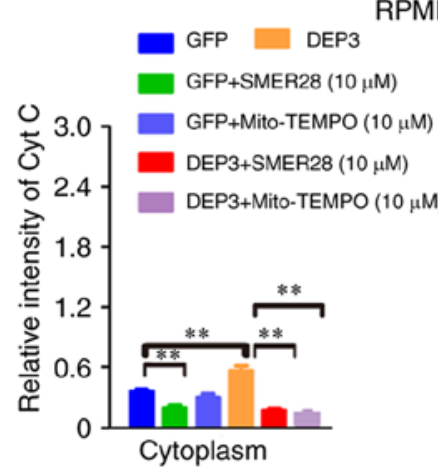

MM.1S

- GFP DEP3

GFP+SMER28 $(10 \mu \mathrm{M})$

- GFP+Mito-TEMPO $(10 \mu \mathrm{M})$
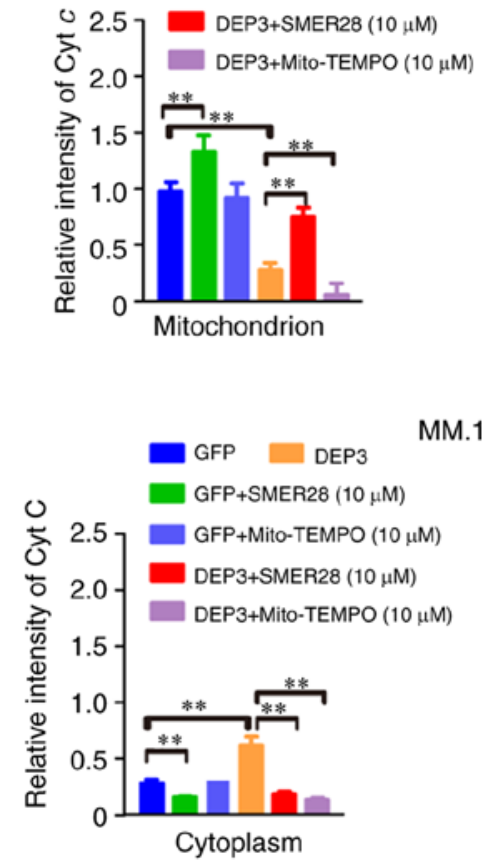

RPMI 8226

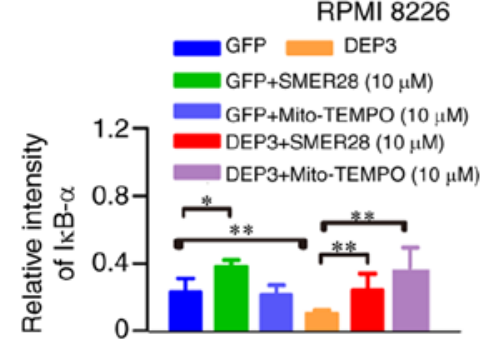




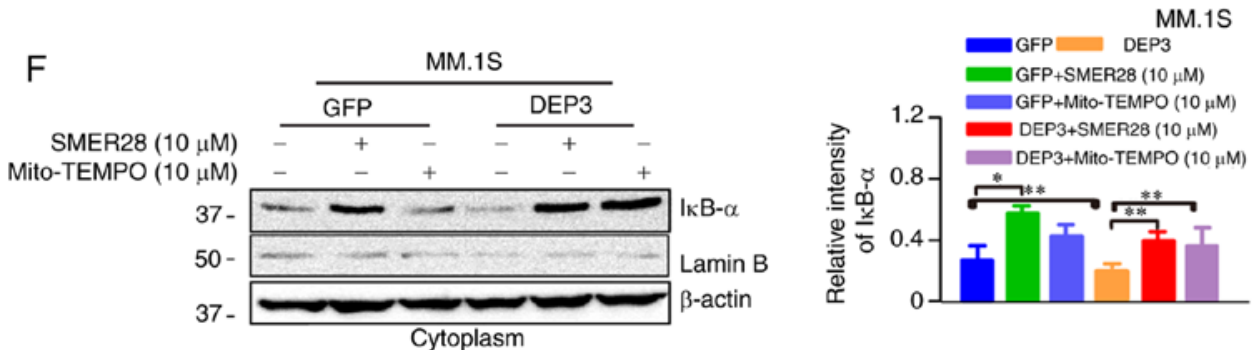

G

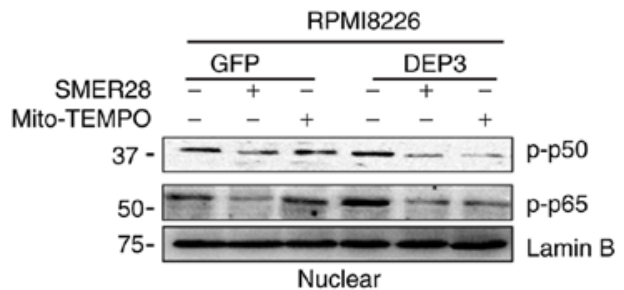

।

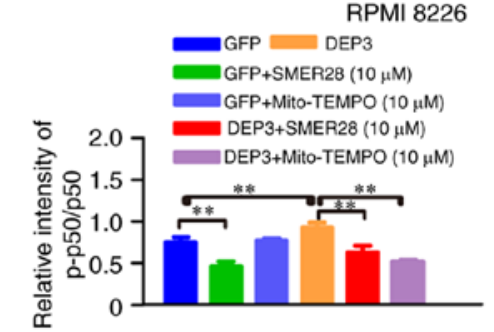

$J$
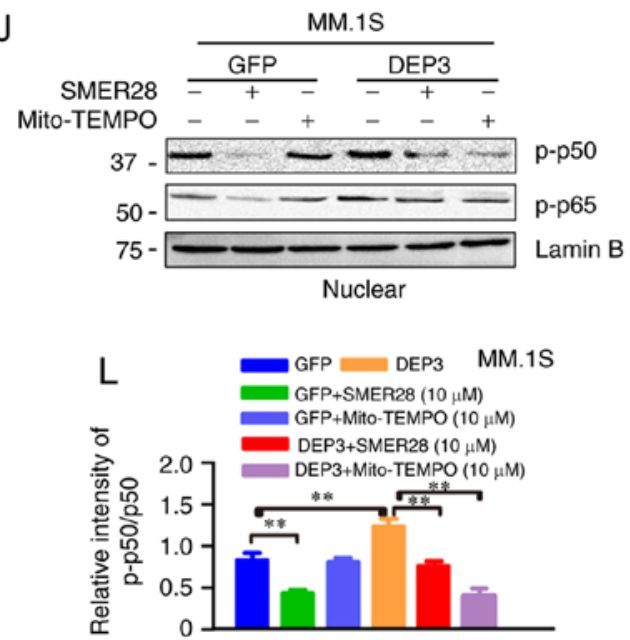

$\mathrm{H}$

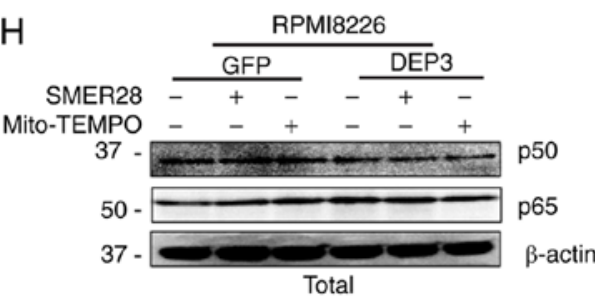

RPMI 8226

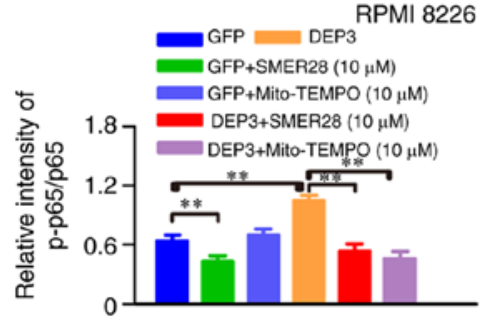

K

K MM.1S

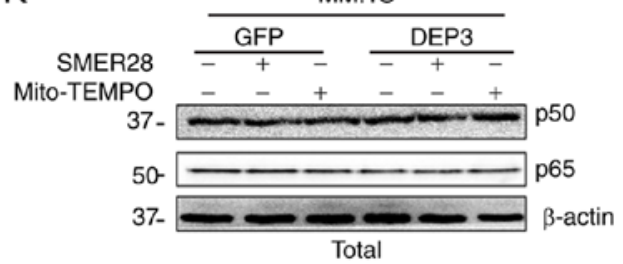

MM.1S

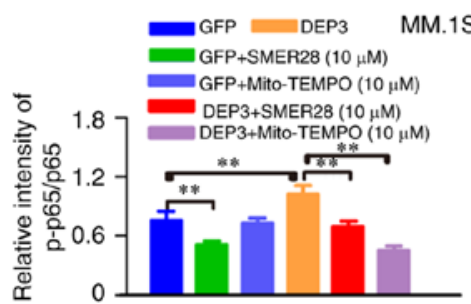

Figure 6. Autophagy inducer and mitochondrial-specific antioxidants reversed the effects of DEPTOR on the angiogenesis of MM cells. RPMI 8226 and MM.1S cells were transfected with lentivirus DEP3 for 5 days and exposed to SMER28 (10 $\mu \mathrm{M})$ or Mito-TEMPO (10 $\mu \mathrm{M})$ for $24 \mathrm{~h}$. (A-D) The mitochondrial and cytoplasmic fractions of the cultured cells were separated using the Mitochondria/Cytosol Fractionation kit. Protein expression of Cyt $c$ in the (A) mitochondria and (B) cytoplasm of the indicated groups for RPMI8226 cells was examined by western blot analysis, respectively. Primary antibodies with COXIV and $\beta$-actin was used as the mitochondrial and cytosolic loading control, respectively. Protein expression of Cyt $c$ in the (C) mitochondria and (D) cytoplasm of the indicated groups for MM.1S was examined by western blot analysis, respectively. Primary antibodies with COXIV and $\beta$-actin was used as the mitochondrial and cytosolic loading control, respectively. Protein expression levels of DEPTOR and IkB- $\alpha$ in the cytoplasm of the indicated groups for (E) RPMI8226 and (F) MM.1S cells were examined by western blot analysis, respectively. (G-L) The nuclear and cytoplasmic protein fractions of the cultured cells were isolated using the NE-PER ${ }^{\mathrm{TM}}$ Nuclear and Cytoplasmic Extraction Reagents. Protein expressions of $(\mathrm{G})$ p-p50 and p-p65 in the nuclear, $(\mathrm{H})$ p50 and p65 in the whole cell lysates of the indicated groups for RPMI8226 cells was examined by western blot analysis, respectively. LaminB and $\beta$-actin was used as the loading control for the nuclear extracts and the whole cell lysates, respectively. (I) Quantification of p-p50 subunit and p-p65 subunit was normalized for total p50 protein and total p65 protein $(\mathrm{G}$ and $\mathrm{H})$, respectively. Protein expression levels of $(\mathrm{J})$ p-p50 and p-p65 in the nuclear, $(\mathrm{K})$ p50 and p65 in the whole cell lysates of the indicated groups for MM.1S was examined by western blot analysis, respectively. Lamin B and $\beta$-actin was used as the loading controls for the nuclear extracts and the whole cell lysates, respectively. (L) Quantification of p-p50 subunit and p-p65 subunit was normalized for total p50 protein and total p65 protein $(\mathrm{J}$ and $\mathrm{K})$, respectively. Error bars indicate the standard deviation from 3 independent experiments. ${ }^{*} \mathrm{P}<0.05$ compared with GFP group, ${ }^{* *} \mathrm{P}<0.01$ compared with GFP group. MM, multiple myeloma; VEGF, vascular endothelial growth factor; Cyt C, cytochrome C; mtROS, mitochondrial reactive oxygen species; p-p50, phospho-p50; p-p65, phospho-p65.

the mechanisms through which autophagy inhibition influences angiogenesis in MM cells. Autophagy defects led to mitochondrial damage and subsequent ROS accumulation. As shown in Fig. 4A and B, staining with Mito-SOX, a mitochondrial superoxide indicator, revealed that DEPTOR inhibition prominently increased the mtROS levels in the RPMI8226 


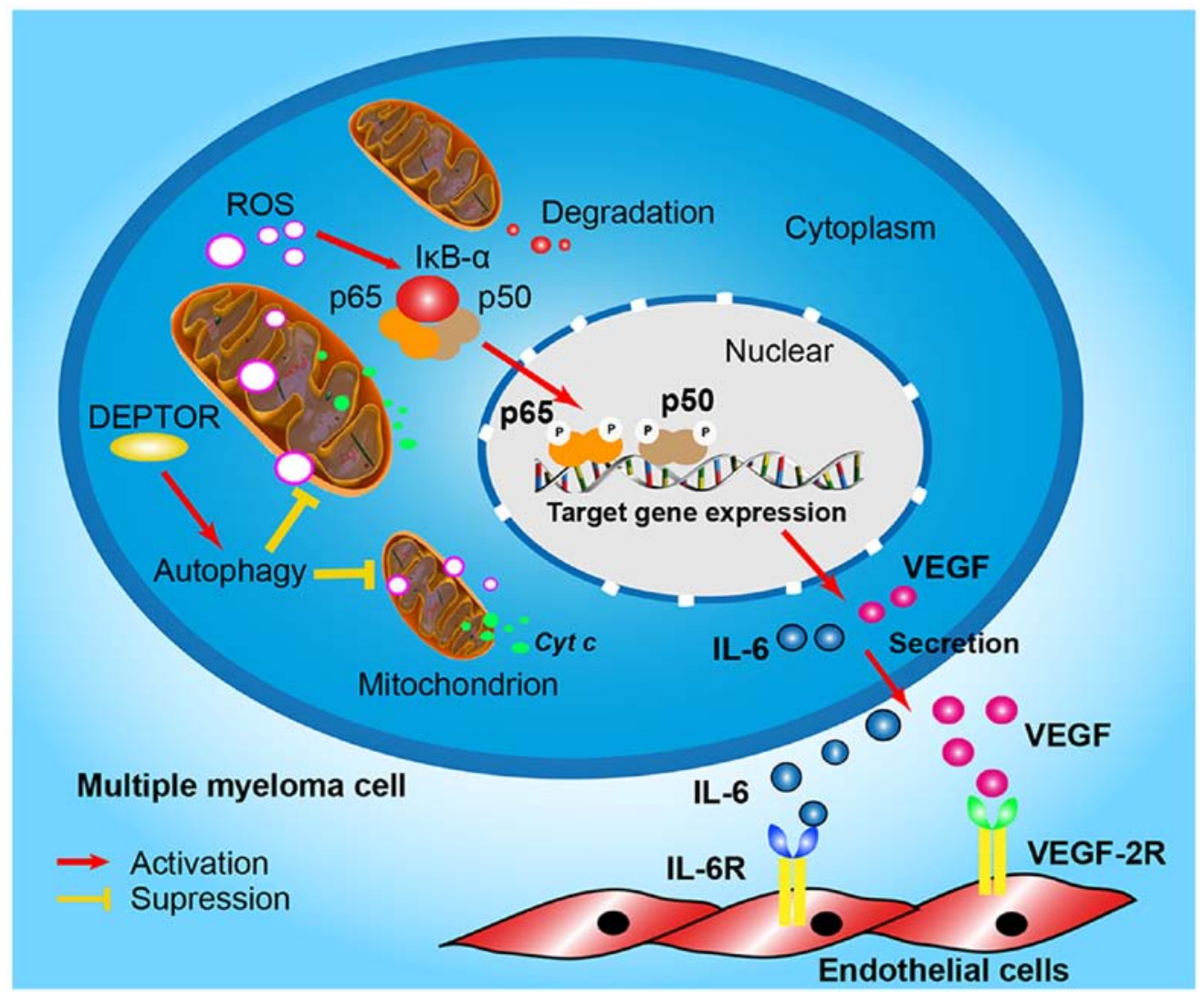

Figure 7. Signaling pathway of DEPTOR regulation of angiogenesis.

and MM.1S cells. As ROS can directly activate the NF- $\kappa \mathrm{B}$ pathway (20), the role of DEPTOR in regulating the NF- $\mathrm{BB}$ pathway was also validated. As shown in Fig. 4C, DEPTOR disruption in RPMI8226 and MM.1S cells stimulated NF- $\kappa$ B activation, as detected by luciferase assays. Western blot analysis demonstrated that DEPTOR inhibition decreased Cyt $c$ expression in the mitochondria and increased Cyt $c$ expression in the cytoplasm, which indicated the potential damage to the mitochondrial membrane of RPMI8226 and MM.1S cells (Fig. 4D-G). Further analyses suggested that DEPTOR disruption decreased I $\kappa \mathrm{B}-\alpha$ expression in the cytoplasm and increased p-p50 and p-p65 expression in the nucleus (Fig. 4H-O). These data revealed that DEPTOR disruption induced mitochondrial damage and the accumulation of mtROS in MM cells, which led to the activation of the NF- $\kappa \mathrm{B}$ pathway.

Autophagy inducer and mitochondrial-specific antioxidant reverse the effects of DEPTOR disruption on autophagy and VEGF expression in MM cells. To confirm the findings described above, the RPMI8226 and MM.1S cells in which DEPTOR was inhibited were treated with SMER28 (an mTOR-independent autophagy inducer) or Mito-TEMPO (a mitochondrial-specific antioxidant) for $24 \mathrm{~h}$. As shown in Fig. 5A, both SMER28 and Mito-TEMPO reversed the effects of DEPTOR disruption on autophagic activity and VEGF expression, but had no effect on DEPTOR expression. When the RPMI8226 and MM.1s cells in which DEPTOR was suppressed were treated with SMER28 or Mito-TEMPO, the detection of the cell supernatant by ELISA revealed that IL-6 and VEGF levels were decreased (Fig. 5B). In addition,
SMER28 and Mito-TEMPO reversed the mitochondrial damage, $m$ tROS accumulation and subsequent $\mathrm{NF}-\kappa \mathrm{B}$ pathway activation induced by DEPTOR disruption (Figs. 5C and 6). These results demonstrate that DEPTOR disruption regulates VEGF by promoting the accumulation of mtROS and activating the $\mathrm{NF}-\kappa \mathrm{B}$ pathway in an autophagy-dependent manner rather than in an mTOR-dependent manner.

\section{Discussion}

DEPTOR is an mTOR-interacting protein and mau be involved in the proliferation, apoptosis, autophagy and differentiation of MM cells $(11,12,17)$. However, whether DEPTOR can regulate the angiogenesis of MM cells has not yet been elucidated. The present study first validated the significance of the DEPTOR mRNA expression level in MM using the Oncomine database. As shown in Fig. 1, compared to the majority of other common cancers, the expression of DEPTOR was significantly increased in MM, which confirmed that DEPTOR plays an important role in the pathogenesis of MM as previously reported $(11,29)$. Based on $3 \mathrm{MM}$ cell lines and primary BM specimens from patients with MM, it was verified that DEPTOR protein expression may negatively regulate the expression of VEGF and MVD in MM. DEPTOR is differentially expressed among patients with MM, and it is required to maintain the differentiation of myeloma cells. A higher expression of DEPTOR may be associated with a better prognosis of patients with MM (29). The overexpression of DEPTOR in MM is associated with the translocation of the MAF transcription factors and the 
CCND1 and CCND3 genes (11), as well as with copy number gains of 8q24 (30). It was hypothesized that the differences in DEPTOR expression may be related to genetic differences among the 3 cell lines. The present study reveals for the first time, to the best of our knowledge, that DEPTOR as an anti-angiogenic factor, negatively correlated with VEGF expression and angiogenesis in patients with MM. Therefore, the potential underlying mechanisms warrant further investigation. The present study used the KEGG-related GSEA analysis to confirm that DEPTOR may be involved in the regulation of MM cells by autophagy, the mTOR signaling pathway and VEGF signaling pathway.

Previous studies have reported that MM patients with a high DEPTOR expression could benefit more from the administration of thalidomide-based therapy than patients with a low DEPTOR expression $(13,14)$. Thalidomide exerts antitumor and anti-angiogenic effects and is currently an important drug for the treatment of MM (31). Accordingly, exploring the mechanisms of DEPTOR in the thalidomide treatment of $\mathrm{MM}$ is essential. In the present study, the results revealed that thalidomide promoted autophagy and DEPTOR expression, but inhibited VEGF expression in MM cells. However, the reduction of DEPTOR expression attenuated the effects of thalidomide on proliferation, apoptosis, autophagy and VEGF expression in MM cells. Notably, in the DEPTOR-disrupted RPMI8226 cells, thalidomide exerted no effects on CRBN, VEGF and autophagy-related proteins. Furthermore, following treatment with thalidomide and 3-MA, the levels of autophagy-related proteins were further inhibited, while VEGF expression was increased. These data suggested that DEPTOR-regulated autophagy may be essential for the antitumor and anti-angiogenic effects of thalidomide, which was independent of the target of thalidomide CRBN. Of note, DEPTOR seems to be associated with a poor prognosis in patients with hepatocellular carcinoma (32) and in some other solid tumors (33), while it is associated with a good prognosis in MM following thalidomide treatment (29). Hence, DEPTOR may be used as a predictor of the efficacy of thalidomide treatment.

Thalidomide treatment can boost the dendritic cell- (34), $\mathrm{T}$ cell- (35) and natural killer cell- (36) mediated immune response in the tumor microenvironment, and inhibit pro-inflammatory cytokine TNF- $\alpha$ production (37). In addition, thalidomide plays an apoptotic role by downregulating Akt activation and triggering caspase 3 activation (38), and induces cell cycle arrest via the upregulation of CDK inhibitor p21 (39). DEPTOR is important for cell proliferation and survival (40). Its upregulation may be the feedback response to thalidomide induced cell apoptosis and cell cycle arrest.

Autophagy is a complex process regulated by different multistep signaling pathways to maintain intracellular homeostasis (41). In the present study, the autophagic regulatory drugs, rapamycin, 3-MA and SMER28, were used to treat MM cells. Rapamycin induces autophagy through the mTOR signaling pathway, while SMER 28 activates autophagy in mTOR-independent manners (42). In the present study, both rapamycin and SMER28 reduced the expression and secretion of IL-6 and VEGF, which proved that autophagy inducers inhibit angiogenesis. The inhibition of autophagy by 3-MA increased the expression, and the secretion of IL-6 and VEGF in MM. Therefore, MM cells can regulate autophagy to modulate angiogenesis via multiple mechanisms. The association of angiogenesis with DEPTOR-mediated autophagy shown in the present study suggests that autophagy plays a role in angiogenesis iin MM.

mtROS, the main source of endogenous ROS, have emerged as essential signal transducers that mediate autophagy (43), which can lead to $\mathrm{NF}-\kappa \mathrm{B}$ signal activation and the secretion of IL-6 and VEGF $(20,21)$. The data presented herein demonstrated that DEPTOR inhibition prominently increased mtROS levels and promoted the production of IL- 6 and VEGF expression through the NF- $\kappa \mathrm{B}$-associated pathway. As DEPTOR is an inhibitor of mTOR, the majority of previous studies are based on the mTOR-dependent pathway $(16,29,44,45)$; however, whether DEPTOR can work independently of mTOR remains unclear (46). In the present study, both non-mTOR-dependent autophagy enhancer (SMER28) and mitochondrial ROS scavenger (Mito-TEMPO) reversed the effects of DEPTOR. These results indicated that DEPTOR can affect angiogenesis through the non-mTOR-dependent autophagy/mtROS pathway rather than through mTOR-dependent pathways. The possible mechanism of angiogenesis regulated by DEPTOR in MM may be that autophagy activation regulated by a high expression of DEPTOR can eliminate excessive ROS produced by the mitochondria, which has effects on cell survival, thereby blocking the NF- $\kappa \mathrm{B}$ signal and downstream molecules IL-6 and VEGF, and inhibiting subsequent angiogenesis. Fig. 7 proposes the pathway of DEPTOR regulation of angiogenesis in MM.

Since DEPTOR is essential for the survival of MM cells, the data of the present study demonstrated that almost all MM cells died following the suppression of DEPTOR, and the effect of DEPTOR cannot be validated in vivo, which was in accordance with previous reports in the literature $(11,29)$. Of note, it seems paradoxical that the knockdown of DEPTOR can lead to death, but increase VEGF expression and angiogenesis, which may promote the development of cancer $(47,48)$. Therefore, the MM cells may preserve their proliferative potential by sacrificing angiogenesis via the high expression of DEPTOR to maintain their own survival. This phenomenon is similar to the role of E-cadherin in breast cancer cells reported in recent literature, in which E-cadherin is also essential for breast cancer cell survival, but at the expense of dissemination and metastasis (49).

The present study is limited by the fact that no immunohistochemistry of DEPTOR was performed. Indeed, MM is not a solid tumor, and the identification of MM cells from bone marrow specimens is difficult. In addition, whether thalidomide treatment can increase the expression of DEPTOR needs to be further confirmed in future clinical studies.

In conclusion, the present study demonstrates that DEPTOR regulates cellular autophagy and mtROS in MM. Angiogenesis may be involved in the process; however, it requires additional study. These results are considered to be important for the further understanding of the novel molecular mechanisms of thalidomide in the treatment of MM.

\section{Acknowledgements}

Not applicable. 


\section{Funding}

The present study was supported by grants from the National Natural Science Foundation of China (no. 81400160 and no. 82070218), the Natural Science Foundation of Fujian Province (no. 2017J01185), the Training Program of Outstanding Young Scientific Researchers of Fujian Universities (no. 2016B027), the Key Personnel Training Project for Young and Middle-Aged Person of Health System of Health and Family Planning Commission of Fujian Province (no. 2016-ZQN-50), and Education and Scientific Research Project for Middle-aged and Young Teachers in Fujian Province (no. JT180185).

\section{Availability of data and materials}

The gene expression data in this study can be found online at the Gene Expression Omnibus under accession numbers GSE24080 (https://www.ncbi.nlm.nih.gov/geo/query/acc. cgi?acc=GSE24080). Data generated or analyzed during this study are available from the corresponding author upon reasonable request.

\section{Authors' contributions}

ZZ and JC conceived the experiment and analyzed data. JW performed the experiments, and generated and collected the data. DQ interpreted the data. JW and ZZ wrote the manuscript. All authors read and approved the final manuscript.

\section{Ethics approval and consent to participate}

All experiments described in the present study were approved by the Ethics Committee of the First Affiliated Hospital of Fujian Medical University. Informed consent was obtained in accordance with the Declaration of Helsinki.

\section{Patient consent for publication}

Not applicable.

\section{Competing interests}

The authors declare that they have no competing interests.

\section{References}

1. Singhal S, Mehta J, Desikan R, Ayers D, Roberson P, Eddlemon P, Munshi N, Anaissie E, Wilson C, Dhodapkar M, et al: Antitumor activity of thalidomide in refractory multiple myeloma. N Engl J Med 341: 1565-1571, 1999.

2. Scott K, Hayden PJ, Will A, Wheatley K and Coyne I: Bortezomib for the treatment of multiple myeloma. Cochrane Database Syst Rev 4: CD010816, 2016.

3. Dimopoulos MA, San-Miguel J, Belch A, White D, Benboubker L, Cook G, Leiba M, Morton J, Ho PJ, Kim K, et al: Daratumumab plus lenalidomide and dexamethasone versus lenalidomide and dexamethasone in relapsed or refractory multiple myeloma: Updated analysis of POLLUX. Haematologica 103: 2088-2096, 2018.

4. Cao Y, Wan N, Liang Z, Xie J, Wang S, Lin T, Zhang T and Jiang J: Treatment outcomes in patients with newly diagnosed multiple myeloma who are ineligible for stem-cell transplantation: Systematic review and network meta-analysis. Clin Lymphoma Myeloma Leuk 19: e478-e488, 2019.
5. Zeng Z, Lin J and Chen J: Bortezomib for patients with previously untreated multiple myeloma: A systematic review and meta-analysis of randomized controlled trials. Ann Hematol 92: 935-943, 2013.

6. André T, Kotelevets L, Vaillant JC, Coudray AM, Weber L, Prévot S, Parc R, Gespach C and Chastre E: Vegf, Vegf-B, Vegf -C and their receptors KDR, FLT-1 and FLT-4 during the neoplastic progression of human colonic mucosa. Int J Cancer 86: 174-181, 2000.

7. Kurebayashi J, Otsuki T, Kunisue H, Mikami Y, Tanaka K, Yamamoto S and Sonoo H: Expression of vascular endothelial growth factor (VEGF) family members in breast cancer. Jpn J Cancer Res 90: 977-981, 1999.

8. Decaussin M, Sartelet H, Robert C, Moro D, Claraz C, Brambilla $\mathrm{C}$ and Brambilla E: Expression of vascular endothelial growth factor (VEGF) and its two receptors (VEGF-R1-Flt1 and VEGF-R2-Flk1/KDR) in non-small cell lung carcinomas (NSCLCs): Correlation with angiogenesis and survival. J Pathol 188: 369-377, 1999.

9. Jussila L, Valtola R, Partanen TA, Salven P, Heikkilä P, Matikainen MT, Renkonen R, Kaipainen A, Detmar M, Tschachler E, et al: Lymphatic endothelium and Kaposi's sarcoma spindle cells detected by antibodies against the vascular endothelial growth factor receptor-3. Cancer Res 58: 1599-1604, 1998.

10. Vacca A, Ribatti D, Roncali L, Ranieri G, Serio G, Silvestris F and Dammacco F: Bone marrow angiogenesis and progression in multiple myeloma. Br J Haematol 87: 503-508, 1994.

11. Peterson TR, Laplante M, Thoreen CC, Sancak Y, Kang SA, Kuehl WM, Gray NS and Sabatini DM: DEPTOR is an mTOR inhibitor frequently overexpressed in multiple myeloma cells and required for their survival. Cell 137: 873-886, 2009.

12. Zhao Y, Xiong X and Sun Y: DEPTOR, an mTOR inhibitor, is a physiological substrate of $\mathrm{SCF}(\beta \operatorname{TrCP}) \mathrm{E} 3$ ubiquitin ligase and regulates survival and autophagy. Mol Cell 44: 304-316, 2011.

13. de la Rubia J and Such E: DEPTOR expression and response to thalidomide: Toward a new therapeutic target in multiple myeloma? Leuk Lymphoma 51: 1960-1961, 2010.

14. Boyd KD, Walker BA, Wardell CP, Ross FM, Gregory WM, Davies FE and Morgan GJ: High expression levels of the mammalian target of rapamycin inhibitor DEPTOR are predictive of response to thalidomide in myeloma. Leuk Lymphoma 51: 2126-2129, 2010.

15. Bruneau S, Nakayama H, Woda CB, Flynn EA and Briscoe DM: DEPTOR regulates vascular endothelial cell activation and proinflammatory and angiogenic responses. Blood 122: 1833-1842, 2013.

16. Ding Y, Shan L, Nai W, Lin X, Zhou L, Dong X, Wu H, Xiao M, Zhou X, Wang L, et al: DEPTOR deficiency-mediated mTORc1 hyperactivation in vascular endothelial cells promotes angiogenesis. Cell Physiol Biochem 46: 520-531, 2018.

17. Zhang H, Chen J, Zeng Z, Que W and Zhou L: Knockdown of DEPTOR induces apoptosis, increases chemosensitivity to doxorubicin and suppresses autophagy in RPMI-8226 human multiple myeloma cells in vitro. Int J Mol Med 31: 1127-1134, 2013.

18. Zhang HR, Chen JM, Zeng ZY and Que WZ: Knockdown of DEPTOR inhibits cell proliferation and increases chemosensitivity to melphalan in human multiple myeloma RPMI-8226 cells via inhibiting PI3K/AKT activity. J Int Med Res 41: 584-595, 2013.

19. Cury PCC, Higashi F, Zacchi FFS, Palhares RB, Quero AA, Dias ALMS, Crusoé EQ and Hungria VTM: Effect of thalidomide on bone marrow angiogenesis in multiple myeloma patients. Hematol Transfus Cell Ther 42: 159-163, 2020.

20. Redza-Dutordoir M and Averill-Bates DA: Activation of apoptosis signalling pathways by reactive oxygen species. Biochim Biophys Acta 1863: 2977-2992, 2016.

21. Liu T, Zhang L, Joo D and Sun SC: NF- $\mathrm{B}$ signaling in inflammation. Signal Transduct Target Ther 2: 17023, 2017.

22. Sun K, Xu L, Jing Y, Han Z, Chen X, Cai C, Zhao P, Zhao X, Yang L and Wei L: Autophagy-deficient Kupffer cells promote tumorigenesis by enhancing mtROS-NF- $\kappa \mathrm{B}-\mathrm{IL} 1 \alpha / \beta$-dependent inflammation and fibrosis during the preneoplastic stage of hepatocarcinogenesis. Cancer Lett 388: 198-207, 2017.

23. Shi L, Campbell G, Jones WD, Campagne F, Wen Z, Walker SJ, Su Z, Chu TM, Goodsaid FM, Pusztai L, et al: The MicroArray Quality Control (MAQC)-II study of common practices for the development and validation of microarray-based predictive models. Nat Biotechnol 28: 827-838, 2010. 
24. Irizarry RA, Hobbs B, Collin F, Beazer-Barclay YD, Antonellis KJ, Scherf U and Speed TP: Exploration, normalization, and summaries of high density oligonucleotide array probe level data. Biostatistics 4: 249-264, 2003.

25. Wang X, Lin Y, Song C, Sibille E and Tseng GC: Detecting disease-associated genes with confounding variable adjustment and the impact on genomic meta-analysis: With application to major depressive disorder. BMC Bioinformatics 13: 52, 2012

26. He W, Fu L, Yan Q, Zhou Q, Yuan K, Chen L and Han Y: Gene set enrichment analysis and meta-analysis identified 12 key genes regulating and controlling the prognosis of lung adenocarcinoma. Oncol Lett 17: 5608-5618, 2019.

27. Rajkumar SV, Dimopoulos MA, Palumbo A, Blade J, Merlini G, Mateos MV, Kumar S, Hillengass J, Kastritis E, Richardson $\mathrm{P}$, et al: International myeloma working group updated criteria for the diagnosis of multiple myeloma. Lancet Oncol 15: e538-e548, 2014

28. Lee N, Lee H, Moon SY, Sohn JY, Hwang SM, Yoon OJ, Youn HS, Eom HS and Kong SY: Adverse prognostic impact of bone marrow microvessel density in multiple myeloma. Ann Lab Med 35: 563-569, 2015

29. Quwaider D, Corchete LA, Misiewicz-Krzeminska I, Sarasquete ME, Pérez JJ, Krzeminski P, Puig N, Mateos MV, García-Sanz R, Herrero AB and Gutiérrez NC: DEPTOR maintains plasma cell differentiation and favorably affects prognosis in multiple myeloma. J Hematol Oncol 10: 92, 2017.

30. Carrasco DR, Tonon G, Huang Y, Zhang Y, Sinha R, Feng B, Stewart JP, Zhan F, Khatry D, Protopopova M, et al: High-resolution genomic profiles define distinct clinicopathogenetic subgroups of multiple myeloma patients. Cancer Cell 9: 313-325, 2006.

31. Mercurio A, Adriani G, Catalano A, Carocci A, Rao L, Lentini G, Cavalluzzi MM, Franchini C, Vacca A and Corbo F: A mini-review on thalidomide: Chemistry, mechanisms of action, therapeutic potential and anti-angiogenic properties in multiple myeloma. Curr Med Chem 24: 2736-2744, 2017.

32. Chen J, Zhu H, Liu Q, Ning D, Zhang Z, Zhang L, Mo J, Du P, Liu X, Song S, et al: DEPTOR induces a partial epithelial-to-mesenchymal transition and metastasis via autocrine TGF $\beta 1$ signaling and is associated with poor prognosis in hepatocellular carcinoma. J Exp Clin Cancer Res 38: 273, 2019.

33. Hu B, Shi D, Lv X, Wu F, Chen S and Shao Z: Prognostic and clinicopathological significance of DEPTOR expression in cancer patients: A meta-analysis. Onco Targets Ther 11: 5083-5092, 2018.

34. Reddy N, Hernandez-Ilizaliturri FJ, Deeb G, Roth M, Vaughn M, Knight J, Wallace P and Czuczman MS: Immunomodulatory drugs stimulate natural killer-cell function, alter cytokine production by dendritic cells, and inhibit angiogenesis enhancing the anti-tumour activity of rituximab in vivo. Br J Haematol 140 36-45, 2008.

35. Haslett PA, Corral LG, Albert M and Kaplan G: Thalidomide costimulates primary human $\mathrm{T}$ lymphocytes, preferentially inducing proliferation, cytokine production, and cytotoxic responses in the CD8+ subset. J Exp Med 187: 1885-1892, 1998.

36. Chang DH, Liu N, Klimek V, Hassoun H, Mazumder A, Nimer SD, Jagannath S and Dhodapkar MV: Enhancement of ligand-dependent activation of human natural killer T cells by lenalidomide: Therapeutic implications. Blood 108: 618-621, 2006.
37. Sampaio EP, Sarno EN, Galilly R, Cohn ZA and Kaplan G: Thalidomide selectively inhibits tumor necrosis factor alpha production by stimulated human monocytes. J Exp Med 173: 699-703, 1991.

38. Mitsiades N,Mitsiades CS, Poulaki V, Chauhan D, Richardson PG, Hideshima T, Munshi NC, Treon SP and Anderson KC: Apoptotic signaling induced by immunomodulatory thalidomide analogs in human multiple myeloma cells: Therapeutic implications. Blood 99: 4525-4530, 2002

39. Escoubet-Lozach L, Lin IL, Jensen-Pergakes K, Brady HA, Gandhi AK, Schafer PH, Muller GW, Worland PJ, Chan KW and Verhelle D: Pomalidomide and lenalidomide induce p21 WAF-1 expression in both lymphoma and multiple myeloma through a LSD1-mediated epigenetic mechanism. Cancer Res 69: 7347-7356, 2009.

40. Hu Y, Su H, Liu C, Wang Z, Huang L, Wang Q, Liu S, Chen S, Zhou J, Li P, et al: DEPTOR is a direct NOTCH1 target that promotes cell proliferation and survival in T-cell leukemia. Oncogene 36: 1038-1047, 2017.

41. Khandia R, Dadar M, Munjal A, Dhama K, Karthik K, Tiwari R, Yatoo MI, Iqbal HMN, Singh KP, Joshi SK and Chaicumpa W: A comprehensive review of autophagy and its various roles in infectious, non-infectious, and lifestyle diseases: Current knowledge and prospects for disease prevention, novel drug design, and therapy. Cells 8: 674, 2019.

42. Koukourakis MI, Giatromanolaki A, Fylaktakidou K, Sivridis E, Zois CE, Kalamida D, Mitrakas A, Pouliliou S, Karagounis IV, Simopoulos K, et al: SMER28 is a mTOR-independent small molecule enhancer of autophagy that protects mouse bone marrow and liver against radiotherapy. Invest New Drugs 36: 773-781, 2018.

43. Roca-Agujetas V, de Dios C, Lestón L, Mari M, Morales A and Colell A: Recent insights into the mitochondrial role in autophagy and its regulation by oxidative stress. Oxid Med Cell Longev 2019: 3809308, 2019.

44. Zhao L, Wang X, Yu Y, Deng L, Chen L, Peng X, Jiao C, Gao G, Tan X, Pan W, et al: OTUB1 protein suppresses mTOR complex 1 (mTORC1) activity by deubiquitinating the mTORC1 inhibitor DEPTOR. J Biol Chem 293: 4883-4892, 2018.

45. Wang Q, Zhou Y, Rychahou P, Harris JW, Zaytseva YY, Liu J, Wang C, Weiss HL, Liu C, Lee EY and Evers BM: Deptor is a novel target of $\mathrm{Wnt} / \beta$-catenin/c-Myc and contributes to colorectal cancer cell growth. Cancer Res 78: 3163-3175, 2018.

46. Catena V and Fanciulli M: Deptor: Not only a mTOR inhibitor. J Exp Clin Cancer Res 36: 12, 2017.

47. Jakob C, Sterz J, Zavrski I, Heider U, Kleeberg L, Fleissner C, Kaiser M and Sezer O: Angiogenesis in multiple myeloma. Eur J Cancer 42: 1581-1590, 2006.

48. Khan MA, Assiri AM and Broering DC: Complement and macrophage crosstalk during process of angiogenesis in tumor progression. J Biomed Sci 22: 58, 2015.

49. Padmanaban V, Krol I, Suhail Y, Szczerba BM, Aceto N, Bader JS and Ewald AJ: E-cadherin is required for metastasis in multiple models of breast cancer. Nature 573: 439-444, 2019.

This work is licensed under a Creative Commons Attribution-NonCommercial-NoDerivatives 4.0 International (CC BY-NC-ND 4.0) License. 